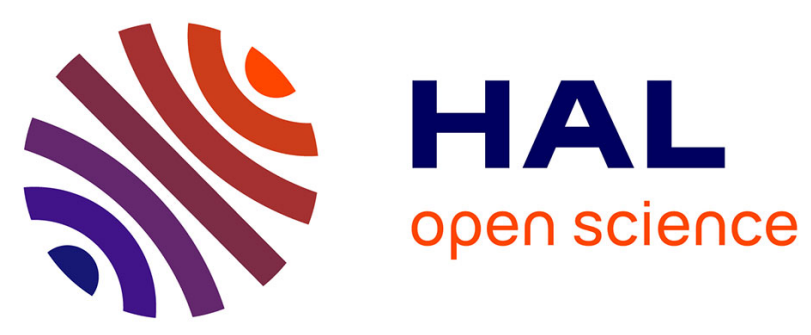

\title{
Anisotropy and shape optimal design of shells by the polar-isogeometric approach
}

Félix D. Kpadonou, Christian Fourcade, Paul de Nazelle, Paolo Vannucci

\section{To cite this version:}

Félix D. Kpadonou, Christian Fourcade, Paul de Nazelle, Paolo Vannucci. Anisotropy and shape optimal design of shells by the polar-isogeometric approach. Journal of Optimization Theory and Applications, 2020, 184 (1), pp.61-97. 10.1007/s10957-018-1425-Z . hal-01818637v3

\section{HAL Id: hal-01818637 https://hal.science/hal-01818637v3}

Submitted on 11 Feb 2019

HAL is a multi-disciplinary open access archive for the deposit and dissemination of scientific research documents, whether they are published or not. The documents may come from teaching and research institutions in France or abroad, or from public or private research centers.
L'archive ouverte pluridisciplinaire HAL, est destinée au dépôt et à la diffusion de documents scientifiques de niveau recherche, publiés ou non, émanant des établissements d'enseignement et de recherche français ou étrangers, des laboratoires publics ou privés. 


\title{
Anisotropy and shape optimal design of shells by the polar-isogeometric approach
}

\author{
D. F. Kpadonou \\ Laboratoire de Mathématiques de Versailles, UMR 8100 \\ Université de Versailles (F) \\ felix.kpadonou@ens.uvsq.fr \\ C. Fourcade \\ Renaut SAS - Guyancourt (F) \\ christian.fourcade@renault.com \\ P. de Nazelle \\ Institut de Recherche Technologique SystemX - Palaiseau (F) \\ paul.denazelle@irt-systemx.fr \\ P. Vannucci \\ Laboratoire de Mathématiques de Versailles, UMR 8100 \\ Université de Versailles (F) \\ paolo.vannucci@uvsq.fr \\ Corresponding author \\ January 20, 2019 \\ https://doi.org/10.1007/s10957-018-1425-z
}

\begin{abstract}
We focus in this paper on the simultaneous shape and material optimal design of shells by an isogeometric approach of a new kind, in which geometry and material properties of the structure are defined by B-spline functions and the design parameters are the control points coordinates. Different kinds of constraints on the regularity, the admissibility of the elastic moduli etc, are taken into account and some numerical examples proving the effectiveness of the approach are given.
\end{abstract}

Key words: Shell design Shape Anisotropy Polar formalism Isogeometric method Naghdi's shell

\section{Introduction}

Structural optimization is a subdomain of optimization that is concerned with the best design of structures, like those of a bridge, an aircraft, a car, a sport device and so on.

The objective of a structural optimization problem can be of different kinds, such as the maximization of stiffness or strength, the minimization of the weight (a typical objective 
in aircraft construction), the maximization of vibration frequencies or of buckling loads and so on.

Apart the objective, normally some constraints are needed to well define the optimization problem; for instance, the maximization of the stiffness is meaningless without a constraint limiting the mass of the structure. A typical constraint entering structural optimization problems is the state equation to be satisfied by any admissible design so by the optimal one too. In structural mechanics, the state equation is actually the equilibrium equation of the system.

Structural optimization problems can be of different types: shape optimization is concerned with the best shape of a structure, e.g. of a beam [1]; topological optimization considers the optimal distribution of the matter inside a given volume, for prescribed boundary conditions and applied forces [2,3]. A more recent type is that concerning the optimization of the material properties $[4,5]$. This branch of structural optimization concerns composite materials and more specifically laminated structures. In such a kind of problems, the best elastic and/or strength properties are to be found, i.e. the mechanical characteristics of the meta-material (i.e. of the laminate) must be designed [6-11]. A particular type of this class of problems is concerned with the best distribution of the local properties of a laminate. In such a case, the elastic moduli of the anisotropic laminate can vary pointwise and their best distribution is the purpose of the optimization problem. In these problems, hence, one has to find the best distribution of one or more tensor fields [12-15].

In this paper, we deal with a new kind of structural optimization problem: the simultaneous optimization of the shape and of the material properties distribution for a shell-like structure.

The purpose of the optimization problem considered in this work is the maximization of the shell stiffness, to be obtained acting on both its shape and the distribution of its elastic anisotropic properties, that can vary pointwise. This is hence a combination of shape and material optimization. Some constraints, concerning namely the mass of the shell or the mechanical admissibility of the elastic moduli, are part of the problem. This kind of study, besides being interesting "per se", could be a contribution to the understanding of how geometry and distributed anisotropy interact, a topic subject of very few studies, see e.g. [16].

The approach that we have used in this paper is an isogeometric-like one. The word isogeometric refers, usually, to numerical techniques in which the solution, or a quantity of interest, of a given problem is discretized using the basis functions describing the exact geometry in an isoparametric sense. Introduced by T.R. Hugues [17], these methods were first implemented in the frame of structural and computational fluid dynamics [1820].

Extensive research has recently been devoted to the isogeometric method, whose principle is based on a direct integration of numerical analysis, optimization and design process in the same environment. The design variables are the control points associated to the Bsplines or NURBS (Non-Uniform Rational Basis Splines) functions used to parameterize the shape of the structure and sometimes their weights [21,22]. The present research follows previous works done in the same direction, [23,24]; the most important innovation 
presented here is the design of the anisotropy properties fields jointly to the design of the optimal shell shape, that is done using the same isogeometric-like technique used for the parameterization of the shell shape. This technique, developed during the PhD thesis [25], is contemporary to the first two published studies using jointly polar parameters and spline parametrization of the design variables, [26,27]. However, unlike these papers, where just the elastic properties are optimized for a fixed geometry, a plate, in our study the design concerns at the same time the elastic properties and the shell shape.

The word isogeometric is normally reserved to approaches where the interpolation functions for the elements representing the structure in a finite element approach are also B-splines or NURBS. This is not the case in our study, where a standard finite element formulation has been used. However, we precise that in this paper the structure behavior is defined by the classical Nagdhi's model (deep shell model written in curvilinear coordinates), so that the state equations of the optimization problem are set up on the domains of charts which define the geometry. Since the three-dimensional structure is parametrized by standard CAD functions, the Naghdi's equations are posed on a square or on a triangle and constitute (through the first and second fundamental forms) an isogeometric-like mechanical interpolation of the structure. Note moreover that, compared with the current industrial standards, this approach of the shape optimization is naturally interfaced with CAD software and allows to simplify the classical optimization process by eliminating the re-meshing steps and the phases of conversion of geometries (from FEM models to CAD models) which deteriorate the optimization results and require dedicated software. For these reasons, we have used the term isogeometric-like to denote our approach, sometimes, for the sake of shortness, simply reduced to isogeometric, in the sense specified above, and, in the end, we have called polar-isogeometric approach the technique presented in this paper, as based, on one side, on the polar formalism and, on the other side, on an isogeometric-like method.

The paper is organized as follows: Section 2 focuses, after a brief recall of the general setting of the considered optimization problem, on the description of the shell model equation governing the behavior of the structure. Section 3 describes the parameterization used for the geometry in the standard isogeometric framework. In Section 4 we describe the polar formalism technique used to represent the elastic tensor. The Section ends with the parametrization used to represent these polar parameters; moreover, we introduce some sufficient conditions to be satisfied by the control points of such parameters in order to ensure the pointwise satisfaction of the admissibility constraints on the elastic tensor. In Section 5 we give the formulation of the optimization problem in the polar-isogeometric framework. The design variables are the control points driving the geometry and the polar parameters. Section 6 presents some numerical examples concerning the optimal design of anisotropic shell structures. The paper ends with a conclusion and an outlook on possible future developments. 


\section{Definition of the Optimization Problem}

\subsection{General Setting of the Design Problem}

A shell is a structure with one dimension (the thickness) small in regard to its other characteristic dimensions, so as it can be identified to a finite surface, generally chosen to be its middle-surface, denoted in the following by $\Omega$. We consider here anisotropic shells, like those constituted of composite materials, and in particular, referring to the recent additive manufacturing technologies of fiber placement, we focus on shells whose anisotropic elastic properties can vary pointwise.

Assuming that the shell thickness is fixed, we are interested in the optimal design of both shell geometry and material distribution. Hence, the design is denoted by a couple $(\Omega, \mathbb{E})$, where $\Omega \subset \mathcal{E}^{3}$, the ordinary Euclidean space, and $\mathbb{E}$ is the elastic tensor of its constitutive materials which can vary pointwise on $\Omega$. Let us start by assuming that $\boldsymbol{U}$, which will be specified later on, is the displacement field associated to a given applicant design $(\Omega, \mathbb{E})$ of the optimization problem. The displacement $\boldsymbol{U}$ is solution of the state (equilibrium) equation: find $\boldsymbol{U} \in \mathbf{W}$ such that

$$
a(\boldsymbol{U}, \boldsymbol{V})=l(\boldsymbol{V}), \forall \boldsymbol{V} \in \mathbf{W} .
$$

In the above variational problem, see [28], we have assumed that the structure is subjected to a system of loads, and $a$ and $l$ are respectively the bilinear form associated to the strain energy and the linear form of work of the applied loads and $\mathbf{W}$ is the space of admissible displacements.

Here, we consider as optimal design problem the maximization of the structure stiffness; as well known, [29], this is equivalent to the minimization of the compliance, the work done by the applied forces, which, in linear elasticity, is exactly twice the strain energy stocked in the structure (Clapeyron's theorem). So, in the end, the objective functional $j$ can be written in the general classical form

$$
j(\boldsymbol{U} ; \Omega, \mathbb{E})=\frac{1}{2} a(\boldsymbol{U}, \boldsymbol{U})
$$

Let us denote by $\left(\Omega^{*}, \mathbb{E}^{*}\right)$ the optimal design

$$
\left(\Omega^{*}, \mathbb{E}^{*}\right)=\arg \min j(\Omega, \mathbb{E}) \text { s.t. }(\Omega, \mathbb{E}) \in \mathcal{E}_{\text {var }} .
$$

$\mathcal{E}_{\text {var }}:=\mathcal{E}_{\Omega} \times \mathcal{E}_{\mathbb{E}} ; \mathcal{E}_{\Omega}$ is the space of admissible geometries which takes into account the regularity constraint, the boundary conditions specified on the geometry, while $\mathcal{E}_{\mathbb{E}}$ takes into account for the admissibility constraints on the elastic tensor.

Within the isogeometric framework, $\Omega$ is defined as the image of a parametric domain $\omega \in \mathbb{R}^{2}$ throughout the mapping $\boldsymbol{\Phi}$, which in the end means that the mechanical problem is defined on $\omega$, thanks to classical differential geometry quantities [30]. We assume that the domain $\omega$ is fixed, henceforth the problem (3) turns to find the optimal mapping $\boldsymbol{\Phi}^{*}$ and $\mathbb{E}^{*}$, such that $\Omega^{*}=\Phi^{*}(\omega)$.

There are different shell models in the literature, among others the Love's classical theory [31], generalizing to shells the classical Kirchhoff's theory for plates, the Koiter's model 
[32] and the Naghdi's one [33], which is the corresponding for shells of the ReissnerMindlin's [34] model of plates. We have used in our study the Naghdi's model, briefly introduced hereafter.

In the following, unless otherwise specified, Greek indexes range in the set $\{1,2\}$ while Latin indexes in $\{1,2,3\}$. The Einstein's summation convention is systematically used over repeated subscript and superscript. Given a point $M$ in the classical Cartesian space $\mathcal{E}^{3}, \boldsymbol{m}$ denotes its position vector with respect to the origin.

\subsection{Naghdi's Shell Model}

Let us denote by $\vec{a}_{3}$ the unit vector normal to $\Omega, \boldsymbol{\xi}=\left(\xi^{1}, \xi^{2}\right)$ and $\xi^{3}$ respectively the curvilinear coordinates associated to $\Omega:=\Phi(\omega)$ and the normal coordinate throughout its thickness $t$; then

$$
\Omega_{t}=\left\{X \in \mathcal{E}^{3}: \boldsymbol{x}=\boldsymbol{\Phi}(\boldsymbol{\xi})+\xi^{3} \vec{a}_{3}, \quad\left(\boldsymbol{\xi}, \xi^{3}\right) \in \omega \times\left[-\frac{t}{2}, \frac{t}{2}\right]\right\} .
$$

$\Omega$ is assumed to be sufficiently regular so that we can define the covariant vectors $\vec{a}_{\alpha}$

$$
\vec{a}_{\alpha}:=\frac{\partial \Phi}{\partial \xi^{\alpha}}=\Phi_{, \alpha}
$$

The vectors $\vec{a}_{\alpha}$ are linearly independent, i.e $a^{\frac{1}{2}}=\left\|\vec{a}_{1} \wedge \vec{a}_{2}\right\| \neq 0$, hence the normal vector is defined as

$$
\vec{a}_{3}:=\frac{\vec{a}_{1} \wedge \vec{a}_{2}}{\left\|\vec{a}_{1} \wedge \vec{a}_{2}\right\|}
$$

Equivalently, one writes

$$
\vec{a}_{\alpha} \wedge \vec{a}_{\beta}=\epsilon_{\alpha \beta} \vec{a}_{3}
$$

where $\epsilon_{\alpha \beta}$ (resp. $\epsilon^{\alpha \beta}$ ) are the covariant (contravariant) Levi-Cevita symbols on the surface defined as follows

$$
\epsilon_{\alpha \alpha}=0, \epsilon_{12}=-\epsilon_{21}=a^{\frac{1}{2}} \text { and } \epsilon^{\alpha \alpha}=0, \epsilon^{12}=-\epsilon^{21}=a^{-\frac{1}{2}} .
$$

The contravariant vectors $\vec{a}^{i}$, dual of the covariant basis vectors $\vec{a}_{i}$, are defined as

$$
\vec{a}_{i} \cdot \vec{a}^{j}=\delta_{i}^{j}
$$

The covariant and mixt components of the curvature tensor are

$$
b_{\alpha \beta}=\vec{a}_{\alpha, \beta} \cdot \vec{a}_{3} \text { and } b_{\alpha}^{\lambda}=\vec{a}_{, \alpha}^{\lambda} \cdot \vec{a}_{3} .
$$

Given a field $\vec{w}$, we denote by $w_{i}$ (resp. $w^{j}$ ) its covariant (resp. contravariant) coordinates

$$
\vec{w}=w_{j} \vec{a}^{j}=w^{k} \vec{a}_{k} .
$$

Letting $\Gamma_{\alpha \beta}^{\lambda}=\vec{a}_{\alpha, \beta} \cdot \vec{a}^{\lambda}$ be the Christoffels symbols, the derivative $\vec{w}_{, \alpha}$ is defined by

$$
\vec{w}_{, \alpha}=\left(w_{\alpha \mid \beta}-b_{\alpha \beta} w_{3}\right) \vec{a}^{\alpha}+\left(w_{3 \mid \beta}+b_{\beta}^{\sigma} w_{\sigma}\right) \vec{a}_{3},
$$


where the symbol " $\mid \beta$ " denotes the covariant derivative operation with respect to $\xi^{\beta}$ defined below

$$
\begin{aligned}
& w_{\alpha \mid \beta}=w_{\alpha, \beta}-w_{\sigma} \Gamma_{\alpha, \beta}^{\sigma}, \\
& w_{3 \mid \beta}=v_{3, \beta} .
\end{aligned}
$$

Kinematical hypotheses. In the Naghdi's shell theory, [33], the following kinematical assumptions are made: the normal fiber in the reference configuration is a straight line which remains straight after deformation but that can rotate, unlike in the Love's and Koiter's shell models. Hence, the displacement of any point of the three-dimensional shell is defined through the displacement vector of the middle-surface plus a rotation of the normal vector $\vec{a}_{3}$. Let us denote by $\vec{u}$ the displacement of the middle-surface and $\boldsymbol{\Psi}$ the rotation vector of the normal. The displacement of any particle of the shell, of position vector $\boldsymbol{\Phi}\left(\xi^{1}, \xi^{2}\right)+\xi^{3} \vec{a}_{3}$ is defined as

$$
\vec{U}=\vec{u}+\xi^{3} \vec{a}_{3} \wedge \Psi\left(\vec{a}_{3}\right)
$$

$\vec{u}$ represents the displacement of the point $M$, on the middle-surface, of position vector $\boldsymbol{m}=\boldsymbol{\Phi}(\boldsymbol{\xi})$, and $\boldsymbol{\Psi}\left(\vec{a}_{3}\right)$ the rotation vector of the normal fiber at the point $M$.

Let $u_{i}$ be the covariant components of $\vec{u}$, i.e. $\vec{u}=u_{i} \vec{a}^{i}$. The rotation vector of the normal fiber is defined as

$$
\Psi\left(\vec{a}_{3}\right)=\epsilon^{\alpha \beta} s_{\alpha} \vec{a}_{\beta}+\Psi_{3} \vec{a}_{3},
$$

where $s_{\alpha}$ are the covariant components and $\Psi_{3}$ the third component of the rotation vector. Hence, from (14), the displacement vector associated to the three-dimensional shell is

$$
\vec{U}=u_{i} \vec{a}^{i}+\xi^{3} s_{\alpha} \vec{a}^{\alpha}
$$

Indeed, the displacement of any particle of the shell is assumed to be described through five degrees of freedom: three for the displacements and two for the rotations.

Hereafter, we denote by $\boldsymbol{u}:=\left(u_{1}, u_{2}, u_{3}\right)$ and $s:=\left(s_{1}, s_{2}\right)$ respectively the vectors of covariant components of displacement and rotation.

\subsection{The State Equation}

The displacement is solution of the state problem which in variational form (virtual work principle), for the static case, reads:

\section{Problem 2.1: State equation}

Find $[\boldsymbol{u}, \boldsymbol{s}] \in \mathbf{W} \subset\left[H^{1}(\omega)\right]^{5}$ such that

$$
a([\boldsymbol{u}, \boldsymbol{s}],[\boldsymbol{v}, \boldsymbol{r}])=l([\boldsymbol{v}, \boldsymbol{r}]), \text { for all }[\boldsymbol{v}, \boldsymbol{r}] \in \mathbf{W}
$$

$l$ is the virtual work of the applied loads, defined as

$$
l([\boldsymbol{v}, \boldsymbol{r}])=\int_{\omega} f^{i} v_{i} \sqrt{a} d S+\int_{\gamma}\left(N^{i} v_{i}+M^{\alpha} r_{\alpha}\right) d l
$$


$d S$ and $d l$ being respectively the element of area over the parametric domain $\omega$ and the element of length on $\Gamma=\Phi(\gamma), \gamma \in \partial \omega$. The strain energy bilinear form $a(\cdot, \cdot)$ is

$$
\begin{gathered}
a([\boldsymbol{u}, \boldsymbol{s}],[\boldsymbol{v}, \boldsymbol{r}])=\int_{\omega} t\left\{Q^{\alpha \beta \lambda \mu} \gamma_{\lambda \mu}(\boldsymbol{u}) \gamma_{\alpha \beta}(\boldsymbol{v})+\frac{t^{2}}{12} Q^{\alpha \beta \lambda \mu} \chi_{\lambda \mu}([\boldsymbol{u}, \boldsymbol{s}]) \chi_{\alpha \beta}([\boldsymbol{v}, \boldsymbol{r}])+\right. \\
\left.E^{\alpha 3 \beta 3} \gamma_{\beta 3}([\boldsymbol{u}, \boldsymbol{s}]) \gamma_{\alpha 3}([\boldsymbol{v}, \boldsymbol{r}])\right\} \sqrt{a} d S
\end{gathered}
$$

In the previous equations

- $\vec{f}=f^{i} \vec{a}_{i}$ is the force per surface element.

- $\vec{N}=N^{i} \vec{a}_{i}$ and $\vec{M}=\epsilon^{\alpha \beta} M_{\alpha} \cdot \vec{a}_{\beta}$ are respectively the resultant of force and the plane moment along the boundary $\Gamma$.

- $E^{i j k l}$ denote the contravariant components of the elastic tensor $\mathbb{E}$ and, $Q^{\alpha \beta \lambda \mu}$ and $E^{\alpha 3 \lambda 3}$ are respectively the reduce plane and anti-plane parts of the elastic tensor.

- Finally $\gamma_{\alpha \beta}, \chi_{\alpha \beta}$ and $\gamma_{\alpha 3}$ are respectively the covariant components of the membrane, bending and shear strains

$$
\begin{aligned}
& \gamma_{\alpha \beta}(\boldsymbol{u})=\frac{1}{2}\left(u_{\alpha \mid \beta}+u_{\beta \mid \alpha}\right)-b_{\alpha \beta} u_{3} \\
& \chi_{\alpha \beta}([\boldsymbol{u}, \boldsymbol{s}])=\frac{1}{2}\left(s_{\alpha \mid \beta}+s_{\beta \mid \alpha}-b_{\alpha}^{\sigma} d_{\sigma \beta}(\boldsymbol{u})-b_{\beta}^{\sigma} d_{\sigma \alpha}(\boldsymbol{u})\right) \\
& \gamma_{\alpha 3}([\boldsymbol{u}, \boldsymbol{s}])=\frac{1}{2}\left(s_{\alpha}+b_{\alpha}^{\sigma} u_{\sigma}+u_{3, \alpha}\right)
\end{aligned}
$$

where $d_{\lambda \mu}(\boldsymbol{u}):=u_{\lambda \mid \mu}-b_{\lambda \mu} u_{3}$.

\section{Geometry Definition for the Isogeometric-Like Ap- proach}

The isogeometric approach relies on the fact that the basis functions used for the parameterization of the fields of interest are the same as those associated to the parametric geometry in a Computed Aided Design (CAD) environment. These functions are typically Bézier, B-spline and NURBS functions. Splines are a more general form of Bezier functions introduced in [35], which in turn have been generalized by NURBS by adding some weights associated to the different blending functions. The aim of this section is to briefly recall the parameterization with B-spline functions, used in the following; for a deep insight in the matter, the reader may refer to [36].

Basis functions definitions In the isogeometric framework, the parametric domain $\omega$ is generally a unit square, called a patch: $\omega=I^{1} \times I^{2}:=[0,1]^{2}$. Let us note $\boldsymbol{\xi}:=\left(\xi^{1}, \xi^{2}\right)$ the parametric coordinates in $\omega$. Assume that each parametric segment $I^{\alpha}$ is partitioned using a knot vector $\Sigma^{\alpha}$ comprising a non-decreasing sequence of real numbers

$$
\Sigma^{\alpha}=\left\{\xi_{1}^{\alpha}, \xi_{2}^{\alpha}, \cdots, \xi_{n_{\alpha}+d_{\alpha}+1}^{\alpha}\right\}, \text { with } \xi_{k}^{\alpha} \leq \xi_{k+1}^{\alpha},
$$

where $\xi_{i}^{\alpha}, n^{\alpha}$, and $d^{\alpha}$ represent respectively the knot of index $i$, the number of univariate spline basis functions spanning the polynomial space over $I^{\alpha}$ and the polynomial degrees. 
Also $d^{\alpha}+1$ is called the order of the spline functions. The breakpoints are not required to be distinct, their multiplicities define the influence of their respective control points. Let us note $\left\{\hat{\xi}_{i}^{\alpha}\right\}_{i \in\left\{1, \cdots, k^{\alpha}\right\}}$ the distinct breakpoints of $I^{\alpha}$, and denote $m_{i}^{\alpha}$ their respective multiplicities, satisfying

$$
\sum_{i=1}^{k^{\alpha}} m_{i}^{\alpha}=n^{\alpha}+d^{\alpha}+1
$$

Univariate B-spline polynomial. Let $I:=[0,1]$ be the parametric interval partitioned with the knot vector $\Sigma=\left\{\xi_{1}, \xi_{2}, \cdots, \xi_{n+d+1}\right\}$, where $n$ and $d$ are respectively the number of spline basis polynomial functions and their degree. The univariate spline functions denoted $b_{i}^{d}$ are defined recursively from (Cox-de-Boor formula, [37])

$$
b_{i}^{0}(\xi)=\left\{\begin{array}{ll}
1 & \text { if } \xi_{i} \leq \xi \leq \xi_{i+1} \\
0 & \text { otherwise }
\end{array}, i \in\{1, \cdots, n+d\}\right.
$$

and for all $p>0$

$$
b_{i}^{p}(\xi)=\frac{\xi-\xi_{i}}{\xi_{i+p}-\xi_{i}} b_{i}^{p-1}(\xi)+\frac{\xi_{i+p+1}-\xi}{\xi_{i+p+1}-\xi_{i+1}} b_{i+1}^{p-1}(\xi), i \in\{1, \cdots, n+d-p\},
$$

where the convention $\frac{0}{0}=0$ is adopted in (24). The B-spline functions satisfy the following properties

Univariate B-spline properties. Let us consider a knot vector

$$
\Sigma=\left\{\xi_{1}, \xi_{2}, \cdots, \xi_{n+d+1}\right\}
$$

partitioning the interval $I$, and denote $k$ and $m_{i}, i=1: k$ respectively the number of distinct knots and their corresponding multiplicities. The B-spline functions satisfy the following properties

- Non-negativity: $b_{i}^{d}(\xi) \geq 0$ for all $\xi \in I$.

- Local support: $\operatorname{supp}\left(b_{i}^{d}\right)=\left[\xi_{i}, \xi_{i+d+1}\right]$.

- Regularity: the functions $b_{i}^{d}$ are at least of regularity $C^{d-m}, m=\max _{i=1: k} m_{i}$ being the maximum value of multiplicity.

Moreover, in the case of knot vectors such that $m_{1}=m_{k}=d+1$, corresponding to the so-called clamped or open spline, one has the unit partition property

$$
\sum_{i=1}^{n} b_{i}^{d}(\xi)=1 \forall \xi \in I .
$$

Bivariate B-spline polynomials Given two knot vectors $\Sigma^{\alpha}=\left\{\xi_{i}^{\alpha}\right\}_{i=1: n^{\alpha}+d^{\alpha}+1}$ partitioning each parametric interval $I_{\alpha}$ of $\omega$, the corresponding bivariate B-spline polynomials 
of degree $d^{\alpha}$ on the parametric coordinate $\xi^{\alpha}$ are defined as

$$
b_{i j}^{\boldsymbol{d}}(\boldsymbol{\xi})=b_{i}^{d^{1}}\left(\xi^{1}\right) b_{j}^{d^{2}}\left(\xi^{2}\right),
$$

where $\boldsymbol{d}=\left(d^{1}, d^{2}\right)$ and $b_{i}^{d^{\alpha}}$ are the univariate spline basis functions of degree $d^{\alpha}$ along the $\alpha^{\text {th }}$ parametric direction.

The bivariate B-spline functions inherit of the same non-negativity, local support properties from the univariate ones. The local support of a function $b_{i j}^{\boldsymbol{d}}$ is $\operatorname{supp}\left(b_{i j}^{\boldsymbol{d}}\right)=$ $\operatorname{supp}\left(b_{i}^{d^{1}}\right) \times \operatorname{supp}\left(b_{j}^{d^{2}}\right)$ and the unit partition property is obtained for knot vectors satisfying the conditions $m_{1}^{\alpha}=m^{\alpha}{ }_{k^{\alpha}}=d^{\alpha}+1 ;\left\{m_{i}^{\alpha}\right\}_{i=1}^{k^{\alpha}}$ being the multiplicities of the distinct breakpoints. Finally the B-spline functions are of class $C^{r}(\omega)$ with $r=\min _{\alpha=1,2} d^{\alpha}-m^{\alpha}$, with $m^{\alpha}=\max _{i=1: k^{\alpha}} m_{i}^{\alpha}$.

Curve and surface definitions Let us denote by $\mathcal{B}_{1}(\Sigma, d)$ the space of univariate B-spline polynomial functions

$$
\mathcal{B}_{1}(\Sigma, d)=\operatorname{span}\left\{b_{i}^{d}, i \in\{1, \cdots, n\}\right\}
$$

A univariate B-spline function $\phi \in \mathcal{B}_{1}(\Sigma, d)$ is defined as a linear combination of the basis functions $b_{i}^{d}$ with their associated coefficients $\boldsymbol{c}_{i}, i \in\{1, \cdots, n\}$

$$
\phi(\xi):=\sum_{i=1}^{n} \boldsymbol{c}_{i} b_{i}^{d}(\xi) .
$$

For a B-spline curve $\mathcal{C}:=\phi(I)$, the coefficients are the coordinates of the control points, so that for a curve in the Cartesian space $\boldsymbol{c}_{i} \in \mathbb{R}^{3}$.

Let $\mathcal{B}_{2}(\boldsymbol{\Sigma}, \boldsymbol{d})$ denote the bivariate B-spline space defined as

$$
\mathcal{B}_{2}(\boldsymbol{\Sigma}, \boldsymbol{d})=\operatorname{span}\left\{\left(b_{i j}^{\boldsymbol{d}}\right)_{i=1: n^{1}}^{j=1: n^{2}}\right\}
$$

We further assume that the middle-surface is defined through a bivariate function $\boldsymbol{\Phi}$ : $\omega \rightarrow \mathbb{R}^{3}$ of the space $\mathcal{B}_{2}(\boldsymbol{\Sigma}, \boldsymbol{d})$. We denote by $\boldsymbol{p}_{i j}:=\left(x_{i j}, y_{i j}, z_{i j}\right) \in \mathbb{R}^{3}$ the coordinate vector of the control points, i.e.

$$
\boldsymbol{\Phi}(\boldsymbol{\xi}):=\sum_{i, j=1}^{n_{1}, n_{2}} \boldsymbol{p}_{i j} b_{i j}^{\boldsymbol{d}}(\boldsymbol{\xi}) .
$$

Figure 1 shows an example of B-spline surface defined using knot vectors of the form $\Sigma^{\alpha}=\{0,0,0,0,1,1,1,1\}$ and $d=3$. For simplicity reasons, we introduce and use as much as possible a unique index for numbering the control points: $i=i(k, l)$ with $i=$ $(l-1) n_{1}+k$, and $i \in\left\{1, \cdots, n^{12}=n^{1} n^{2}\right\}$. So the function $\Phi$ can be rewritten as

$$
\boldsymbol{\Phi}(\boldsymbol{\xi})=\sum_{i=1}^{n^{12}} \boldsymbol{p}_{i} b_{i}^{\boldsymbol{d}}(\boldsymbol{\xi})
$$




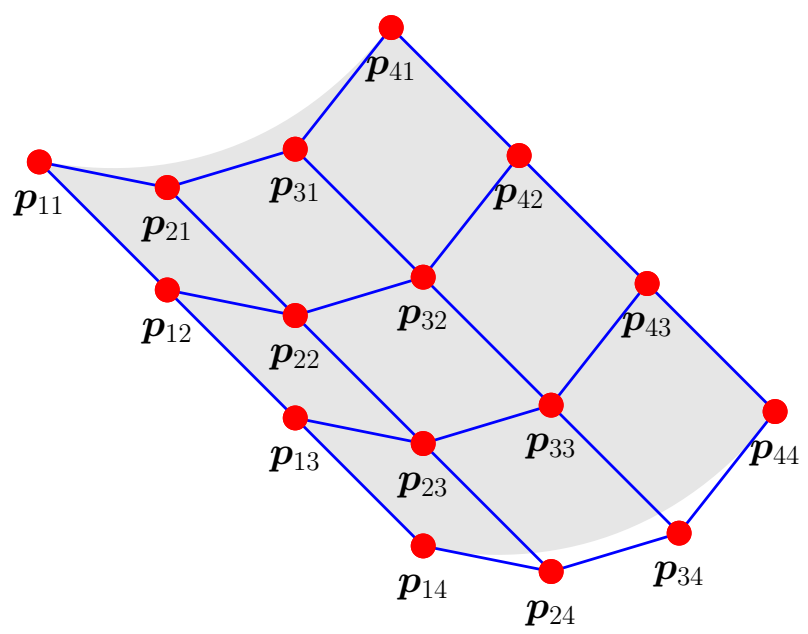

Figure 1: Example of middle-surface with the associated control points

At this point, one remarks that the shape can be driven by the control points $\boldsymbol{p}_{i}$. Moreover, some constraints on the geometry can be stated and handled very easily. For instance, a fix place boundary condition on the edge of parametric boundary $\xi^{1}=0$, thanks to the fact that the knots are assumed open or clamped, turns to keep fixed the control points $\left(\boldsymbol{p}_{1 j}\right)_{j=1}^{n_{2}}$ associated to that edge.

\section{Anisotropy Representation}

An anisotropic material has elastic properties changing with the direction. Such properties are expressed by the elastic tensor. The main goal when designing such materials is to set up the optimal distribution of the elastic properties. In the case of optimal design of planar anisotropic structures, it is suitable to make use of the polar formalism, introduced by Verchery [38] in 1979, to represent a planar elastic tensor using just invariants and angles. This formalism allows to easily represent rotations and the constraints on the design variables (the polar parameters).

Moreover, the polar formalism allows to split the elastic tensor into its isotropic and anisotropic parts; hence it offers the possibility to target and explicitly tune the anisotropy. More details on the polar formalism can be found in [39-41]. For a complete presentation of anisotropic elasticity and of the polar method and its applications, the reader can refer to [5].

The polar formalism has successfully been applied to several different optimization problems concerning laminated structures, [6-16,42-48] as well as to some theoretical problems, [40,41, 49-57].

As said above, the mechanical properties of concern in this study are condensed in the elasticity tensor $\mathbb{E}$; this is a fourth-rank tensor whose components satisfy the minor and major symmetries of the indexes:

$$
E^{i j k l}=E^{j i k l}=E^{i j l k}=E^{k l i j}, \quad i, j, k, l=1,2,3 .
$$


For the case of a plane tensor, which is of interest in our study, as detailed below, the independent elastic components reduce then to only 6: $E^{1111}, E^{1112}, E^{1122}, E^{1212}, E^{1222}$ and $E^{2222}$, in the following generically indicated as $E^{\alpha \beta \lambda \mu}$.

In the polar formalism, the components $E^{\alpha \beta \lambda \mu}$ of the plane elastic tensor are expressed as

$$
\begin{aligned}
& E^{1111}=T_{0}+2 T_{1}+R_{0} \cos 4 \Phi_{0}+4 R_{1} \cos 2 \Phi_{1}, \\
& E^{1112}=R_{0} \sin 4 \Phi_{0}+2 R_{1} \sin 2 \Phi_{1}, \\
& E^{1122}=-T_{0}+2 T_{1}-R_{0} \cos 4 \Phi_{0}, \\
& E^{1212}=T_{0}-R_{0} \cos 4 \Phi_{0}, \\
& E^{1222}=-R_{0} \sin 4 \Phi_{0}+2 R_{1} \sin 2 \Phi_{1}, \\
& E^{2222}=T_{0}+2 T_{1}+R_{0} \cos 4 \Phi_{0}-4 R_{1} \cos 2 \Phi_{1} .
\end{aligned}
$$

In the above equation, $T_{0}, T_{1}, R_{0}$ and $R_{1}$ are elastic moduli, while $\Phi_{0}$ and $\Phi_{1}$ are (the polar) angles. In particular, it can be shown that $T_{0}$ and $T_{1}$ are the isotropy invariants while $R_{0}, R_{1}, \Phi_{0}-\Phi_{1}$ are the anisotropy invariants. The elasticity is then represented through intrinsic quantities, tensor invariants, and angles, which is particularly suitable when working with orientation depending properties, like in anisotropy. The choice of one of the two polar angles fixes the frame: as $\Phi_{0}-\Phi_{1}$ is an invariant, choosing $\Phi_{0}$ or $\Phi_{1}$ corresponds to fix a frame and also the value of the other angle.

It can be shown, see [39], that the polar invariants are linked to the elastic symmetries. In particular, ordinary orthotropy corresponds to the condition

$$
\Phi_{0}-\Phi_{1}=K \frac{\pi}{4} ; \quad K=0,1
$$

The value of $K$ is very important in optimization problems; in fact, it has been seen in several cases that changing $K$ from 0 to 1 or vice-versa transforms an optimal solution into an anti-optimal one (i.e the best to the worst), [44]. Taking into account for (35) in (34), we obtain for an orthotropic layer:

$$
\begin{aligned}
& E^{1111}\left(\Phi_{1}\right)=T_{0}+2 T_{1}+R_{0}^{K} \cos 4 \Phi_{1}+4 R_{1} \cos 2 \Phi_{1}, \\
& E^{1112}\left(\Phi_{1}\right)=-R_{0}^{K} \sin 4 \Phi_{1}-2 R_{1} \sin 2 \Phi_{1}, \\
& E^{1122}\left(\Phi_{1}\right)=-T_{0}+2 T_{1}-R_{0}^{K} \cos 4 \Phi_{1}, \\
& E^{1212}\left(\Phi_{1}\right)=T_{0}-R_{0}^{K} \cos 4 \Phi_{1}, \\
& E^{1222}\left(\Phi_{1}\right)=R_{0}^{K} \sin 4 \Phi_{1}-2 R_{1} \sin 2 \Phi_{1}, \\
& E^{2222}\left(\Phi_{1}\right)=T_{0}+2 T_{1}+R_{0}^{K} \cos 4 \Phi_{1}-4 R_{1} \cos 2 \Phi_{1},
\end{aligned}
$$

with

$$
R_{0}^{K}=(-1)^{K} R_{0}
$$

Two other special orthotropies exist: square symmetry (i.e with elastic properties periodic of $\frac{\pi}{2}$ ), corresponding to the condition $R_{1}=0$ and $R_{0}$-orthotropy, corresponding to $R_{0}=0$. For more details on this subject, the reader is referred to $[39,58]$. 
To summarize, in the polar formalism, the following six parameters define the elastic tensor in any frame:

- two isotropic invariants $T_{0}, T_{1}$;

- three anisotropic invariants $R_{0}, R_{1}, \Phi_{0}-\Phi_{1}$. For ordinarily orthotropic layers, these can be replaced by the two quantities $R_{0}^{K}$ and $R_{1}$, still representing the three invariants (indeed $K \in\{0,1\}$ )

- the angle $\Phi_{1}$, fixing the frame.

We finally remark that isotropy corresponds to $R_{0}=R_{1}=0$.

\subsection{Elastic Assumptions}

In this paper, we consider the optimal design of a shell under the following assumptions: the shell is locally orthotropic everywhere and homogeneous through the thickness. The design concerns exclusively the anisotropic part of $\mathbb{E}$, for both the direction and the elastic moduli.

This is a simplified setting, but it corresponds to a real situation, that of a shell composed by a quasi-homogeneous orthotropic laminate of identical orthotropic plies, see e.g. [59]. In such a case, the elastic behavior of the laminate is completely determined by a unique elastic tensor, describing at the same time the extension and the bending response of the shell, and there is no coupling between extension and bending. In addition, because the plies are identical, the isotropic part, i.e. the polar invariants $T_{0}$ and $T_{1}$, are everywhere equal to those of the basic layer, so they cannot be affected by the design process, once the material chosen. We precise, however, that here we simply address the problem specified hereon, just as a mathematical problem, regardless of whether or not it corresponds to the above laminate; that is why we still use the symbol $\mathbb{E}$ to denote the stiffness tensor.

We also assume that the through-the-thickness properties $E^{\alpha 3 \beta 3}$ are much less important for the process at hand than the in-plane ones, so they are simply considered as constant throughout the design process. This fact is justified for thin shells, as we assume the shells at hand to be; in addition, this approximation is consistent with the fact, always confirmed by the numerical results, that the optimal shell is the one working in a membrane regime, where shear and bending energy tends to zero; as a consequence, the transversal shear moduli $E^{\alpha 3 \beta 3}$ are inessential in this context.

Finally, the main consequence of such assumptions for the optimum design of the shell is that the number of elastic design variables is reduced to only three: $R_{0}^{K}, R_{1}$ and $\Phi_{1}$ : two elastic moduli and the orthotropy direction.

\subsection{Constraints on the Polar Parameters}

The polar elastic moduli cannot take arbitrary values, they are submitted to some constraints that can be of two types, depending upon whether the shell is homogeneous (i.e., composed by a unique layer) or not (i.e., it is a laminated shell): 
- Elastic bounds, see [55], resulting from the positive definiteness of $\mathbb{E}$, [5]:

$$
\begin{aligned}
T_{1}\left[T_{0}+R_{0}^{K}\right] & >2 R_{1}^{2}, \\
T_{0} & >\left|R_{0}^{K}\right|, \\
R_{1} & \geq 0 .
\end{aligned}
$$

Such constraints must be satisfied locally by any elastic homogeneous sheet in a planar elastic state.

- Geometric bounds: it can be shown, see [40], that laminates composed by identical layers cannot realize all the possible combinations of the values of the elastic moduli. We could say, in some words, that laminates form a "more restricted" elastic class. Mathematically speaking, this corresponds to the fact that the bounds on $\mathbb{E}$ are not (38) but some other more restrictive ones, called geometric bounds, because linked to the stacking sequence. For the case of an orthotropic laminate composed of orthotropic layers, such geometric bounds are (here, $K_{L}, R_{0}^{K^{L}}=(-1)^{K_{L}} R_{0}^{L}$ and $R_{1}^{L}$ are polar parameters of the basic layer)

$$
\begin{aligned}
2\left(\frac{R_{1}}{R_{1}^{L}}\right)^{2}-1 & \leq \frac{R_{0}^{K}}{R_{0}^{K L}}, \\
R_{0}^{K} & \leq R_{0}^{L}, \\
0 \leq R_{1} & \leq R_{1}^{L} .
\end{aligned}
$$

Since equation (39) is more restrictive than (38), when the problem concerns the design of a laminated structure, eq. (38) must be replaced by eq. (39), otherwise, one could obtain some values for the components of $\mathbb{E}$ that cannot be realized in practice through a laminate composed by identical plies.

\subsection{Isogeometric Parameterization for the Polar Parameters}

We have to recall now that in the problem at hand, the elastic properties can vary pointwise throughout the shell. This means that the design variables $R_{0}^{K}, R_{1}$ and $\Phi_{1}$ are actually design variable fields, with the constraints (38) or (39) to be satisfied everywhere in the shell. In other words, put in this way the problem is infinitely dimensional with an infinite number of constraints. A discretization of the problem is hence needed, in order to reduce its dimension and the number of constraints to a finite value.

Using a standard finite element based discretization, like in $[12,14,15]$, would yield to a huge number of design variables and of constraints. Following an idea originally proposed in [26], we propose below a new approach, based upon an isogeometric-like parameterization of the polar quantities, in a way similar to that used to parametrize the shell geometry. Just as in [26], but unlike in [27], where only the polar angle is described in terms of an isogeometric surface, here we use a parametrization of the invariants $R_{0}^{K}$ and $R_{1}$, described, like the polar angle, by an isogeometric surface each one, so obtaining a complete optimization of $\mathbb{E}$. The design parameters associated to $\mathbb{E}$ are the polar parameters defined at each control point of the spline functions describing the fields of the 
corresponding polar parameters of $\mathbb{E}$ and we derive below a finite set of sufficient constraints which guarantee the elastic or geometric bounds to be satisfied everywhere in the shell.

As already said, we assume the polar parameters fields to be designed under the form of Bézier, B-spline or NURBS functions. We focus mostly on the parameterizations of the polar moduli $R_{0}^{K}$ are $R_{1}$ which are mainly involved in the elastic and geometric constraints. Indeed, our goal is to define a set of constraints on the control points which ensure that the inequalities (38) are satisfied pointwise. Two parameterizations for $R_{1}$ are relevant (here, we refer to the elastic bounds (38), but a similar procedure can be used also for the case of the geometric bounds (39)):

- a conformal parameterization: $R_{1}$ is parametrized as the square root of a positive Bspline; the interest of this change of variable is to simplify the constraints (38) which become linear. The drawback is that the elastic coefficients and the constraints are no more differentiable at $R_{1}=0$;

- a direct parametrization: $R_{1}$ is parameterized by a B-spline but, see Propostion 4.3, the constraints on the control points depend on the number of control points. However, this gives the advantage of making the elasticity tensor differentiable with respect to the control points of the polar parameters.

On the other hand the basis functions used for the parameterization of $R_{0}^{K}$ must be the same as the ones used for the parameterization of $R_{1}$ or $R_{1}^{2}$. The main results of this part, given in the Proposition 4.2 and 4.3, descend directly from the following Proposition 4.1.

Proposition 4.1. Let $\boldsymbol{\Sigma}=\left(\Sigma^{1}, \Sigma^{2}\right)$ and $\boldsymbol{d}=\left(d^{1}, d^{2}\right)$ be two couples of knot vectors and integer numbers

i) The B-spline basis functions of $\mathcal{B}_{2}(\boldsymbol{\Sigma}, \boldsymbol{d})$ are such that: $\forall i \in\left\{1, \cdots, n^{12}\right\}$ and $n^{12}=$ $n^{1} \times n^{2}$

$$
\forall \boldsymbol{\xi} \in \omega:=\left[\xi_{1}^{1}, \xi_{n^{1}+d^{1}+1}^{1}\right] \times\left[\xi_{1}^{2}, \xi_{n^{2}+d^{2}+1}^{2}\right] \text { and } 0 \leq b_{i}^{\boldsymbol{d}}(\boldsymbol{\xi}) \leq 1 \text {. }
$$

ii) Moreover if the end knot-points are of full multiplicity the B-spline functions satisfy the unit partition property

$$
\forall \boldsymbol{\xi} \in \omega, \sum_{i=1}^{n^{12}} b_{i}^{\boldsymbol{d}}(\boldsymbol{\xi})=1 .
$$

iii) If $C$ is a B-spline in $\mathcal{B}_{2}(\boldsymbol{\Sigma}, \boldsymbol{d})$ of control points $c^{i}$ then the relationships $c^{i} \leq 0$ for all $i$ entail that $C(\boldsymbol{\xi}) \leq 0$ for any $\boldsymbol{\xi} \in \omega$. The Example 4.1 shows that the converse is false.

iv) If $G$ and $H$ are two B-splines in $\mathcal{B}_{2}(\boldsymbol{\Sigma}, \boldsymbol{d})$ then for any $(a, b, c) \in \mathbb{R}^{3}$ the linear combination $S=a G+b H+c$ is a B-spline which belongs to $\mathcal{B}_{2}(\boldsymbol{\Sigma}, \boldsymbol{d})$. Moreover, if $g^{i}$ and $h^{i}$ denote respectively the control points of $G$ and $H$, the control points $s^{i}$ of $S$ are given by

$$
s^{i}=a g^{i}+b h^{i}+c
$$

Proof The proofs of properties $i$ ) and ii) are given in [60]. Property iii) is a consequence 
of the positiveness property of the B-spline basis functions. For the property $i v$ ), we see from the equation (41) that the constant function $c: \boldsymbol{\xi} \mapsto c$ can be written as

$$
c=\sum_{i=1}^{n^{12}} c b_{i}^{\boldsymbol{d}}(\boldsymbol{\xi}) \text { for all } \boldsymbol{\xi} \in \omega
$$

and thus $c$ is a "B-spline function" in $\mathcal{B}_{2}(\boldsymbol{\Sigma}, \boldsymbol{d})$, the result is a consequence of the fact that $\mathcal{B}_{2}(\boldsymbol{\Sigma}, \boldsymbol{d})$ is a vector space.

Remark 4.1. B-splines do not, in general, interpolate the endpoints of the control polygon. Their associated knot vectors define the influence of the control points or parameters. The control polygon will coincide with the B-spline surface at a knot of full multiplicity. We will consider knot vectors with first and last knot breakpoints of full multiplicity with respect to the orders of the B-spline function. This point is necessary for the property (40) in the Proposition 4.1.

It is worth noting, as the following example shows, that the proposed approach yields to sufficient but not necessary constraints.

Example 4.1. Let us consider the knot vector $\Sigma=\{0,0,0,0,1,1,1,1\}$ and assume $d=3$. The polynomial basis functions are the classical Bernstein functions

$$
b_{1}^{3}(\xi)=(1-\xi)^{3}, \quad b_{2}^{3}(\xi)=3(1-\xi)^{2} \xi, \quad b_{3}^{3}(\xi)=3(1-\xi) \xi^{2}, \quad b_{4}^{3}(\xi)=\xi^{3} .
$$

In this case 0 and 1 are break-points of full multiplicity and the curve $C_{p}$ interpolates these endpoints which are below the horizontal line representing the zero ordinate line. However, as can be noticed on Figure 2, the control points $\boldsymbol{p}_{1}$ and $\boldsymbol{p}_{2}$ do not satisfy the negativity constraint while the value of the spline polynomial is always negative.

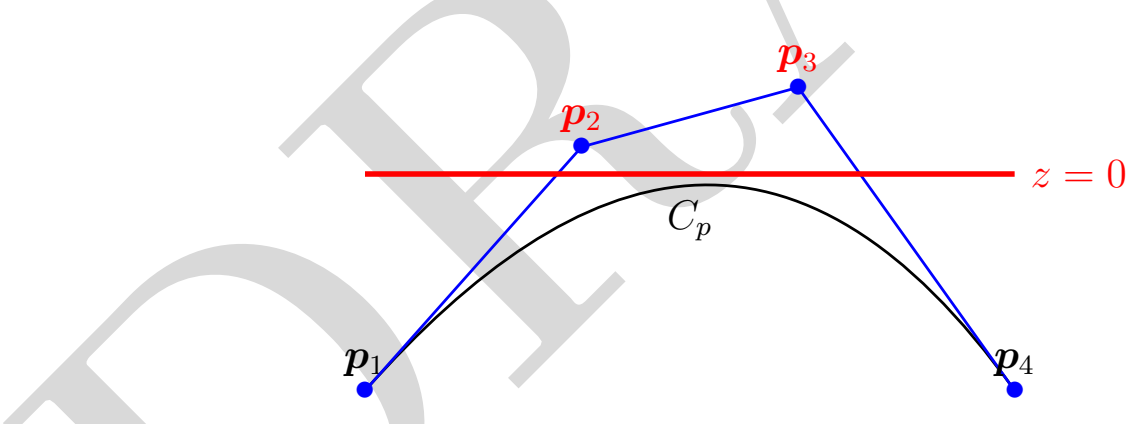

Figure 2: Example of clamped B-spline. In this particular case, the control points: $\boldsymbol{p}_{1}=-2.0, \boldsymbol{p}_{2}=0.25$, $\boldsymbol{p}_{3}=0.8$ and $\boldsymbol{p}_{4}=-2.0$ are not all negative while $C(\xi)<0$ for all $\xi \in[0,1]$.

\subsection{Constraints for Conformal Parameterization}

In this case, the polar parameters $R_{0}^{K}$ and $R_{1}^{2}$ are parametrized by B-splines and we show in the Proposition 4.2 that this allows to reduce the non-linear constraints (38) to some linear constraints set on the control points.

Proposition 4.2. Let $\boldsymbol{d}=\left(d^{1}, d^{2}\right)$ and $\boldsymbol{\Sigma}=\left(\Sigma^{1}, \Sigma^{2}\right)$ be two pairs of integers and knotvectors. Assume that $\mathcal{R}$ and $\mathcal{R}_{0}$ are two $B$-spline parameterizations of $R_{1}^{2}$ and $R_{0}^{K}$ in 
$\mathcal{B}_{2}(\boldsymbol{\Sigma}, \boldsymbol{d})$, written as

$$
\mathcal{R}(\boldsymbol{\xi})=\sum_{i=1}^{n^{12}} r^{i} b_{i}^{\boldsymbol{d}}(\boldsymbol{\xi}), \mathcal{R}_{0}(\boldsymbol{\xi})=\sum_{i=1}^{n^{12}} r_{0}^{i} b_{i}^{\boldsymbol{d}}(\boldsymbol{\xi})
$$

where $n^{\alpha}=\operatorname{card}\left(\Sigma^{\alpha}\right)-d^{\alpha}-1(\alpha=1,2)$ and $n^{12}=n^{1} \times n^{2}$. If the following inequalities

$$
\begin{aligned}
T_{1}\left[T_{0}+r_{0}^{i}\right] & >2 r^{i}, \\
-T_{0} & <r_{0}^{i}<T_{0}, \\
r^{i} & \geq 0
\end{aligned}
$$

are satisfied $\forall i \in\left\{1, \cdots, n^{12}\right\}$, then the inequalities (38) are satisfied for all $\boldsymbol{\xi} \in \omega$.

In an analogous way, for the typical case of laminate made of identical orthotropic layers, the constraints for the geometric bounds (39) are

$$
\begin{aligned}
2 \frac{r^{i}}{\left(R_{1}^{L}\right)^{2}}-1 & \leq \frac{r_{0}^{i}}{R_{0}^{K}} \\
r_{0}^{i} & \leq R_{0}^{L} \\
0 \leq r^{i} & \leq\left(R_{1}^{L}\right)^{2}
\end{aligned}
$$

with $R_{0}^{K L}, R_{1}^{L}$ the polar parameters of the basic orthotropic layer, defined in Sec. 4.

\subsection{Constraints for Direct Parameterization}

We now assume that the polar parameter $R_{1}$ is parametrized by a $\mathrm{B}$-spline. Consider the case of elastic bounds, this new parameterization only affects the inequality $(38)_{1}$. We have the following result:

Proposition 4.3. Let $\mathcal{R}_{1}$ and $\mathcal{R}_{0}$ be two $B$-spline parametrizations of the polar moduli $R_{1}$ and $R_{0}^{K}$, written as:

$$
\mathcal{R}_{1}(\boldsymbol{\xi})=\sum_{i=1}^{n^{12}} r_{1}^{i} b_{i}^{\boldsymbol{d}}(\boldsymbol{\xi}) \quad \text { and } \quad \mathcal{R}_{0}(\boldsymbol{\xi})=\sum_{i=1}^{n^{12}} r_{0}^{i} b_{i}^{\boldsymbol{d}}(\boldsymbol{\xi})
$$

Then the inequalities

$$
2\left(r_{1}^{i}\right)^{2}-\frac{T_{1}\left(T_{0}+r_{0}^{i}\right)}{n^{12}}<0, \quad \forall i \in\left\{1, \cdots, n^{12}\right\}
$$

imply the inequality $(38)_{1}$ to be satisfied for every $\boldsymbol{\xi} \in \omega$.

In the case of geometric bounds on the elastic tensor, the direct parameterization in regard to the conformal one, affects the inequalities $(39)_{1}$ and $(39)_{2}$ : the sufficient inequalities to 
be satisfied, respectively for $(39)_{1}$ and $(39)_{2}$ are then

$$
\begin{aligned}
2\left(\frac{r_{1}^{i}}{R_{1}^{L}}\right)^{2}-\frac{1}{n^{12}}\left(1+\frac{r_{0}^{i}}{R_{0}^{K^{L}}}\right) & \leq 0 \\
0 \leq r_{1}^{i} & \leq R_{1}^{L}
\end{aligned}
$$

Reader may refer to [25] for the proof which is based on B-spline properties and the use of the Schwartz inequality.

The parameterizations stated above for the polar parameters allow to reduce the number of design variables which are now the control points of their parameterizing B-spline functions. But Figure 2 of the Example 4.1 shows that defining the constraints directly on the control points can lead to a reduction of the design space exploration.

Nevertheless, it is possible to use B-spline flexibility to define the constraints on new control points in order to enlarge the admissible space exploration. More specifically, once the parametrizations of the polar parameters are set, one can use subdivision (through Casteljau algorithm) see [61] or knot insertion flexibility of B-spline to define some new "more interpolating" control points, $[36,60]$. By doing so, we can define new control parameters which are linear combinations of the original control points and more suitable for the definition of more accurate constraints. This yields to "relax" the bounds of variation on the original design variables (control points) and allows to enlarge the admissible space exploration. The constraints will then be checked on the new control points. This strategy is shown in the following Example.

Example 4.2. (B-spline flexibility for the constraints). Let us return to the Example 4.1. Figure 3 shows the spline curve with its initial control points $p_{i}$ and some news ones $l_{i}$ and $r_{j}$, which are associated to the two splines obtained by inserting an interpolant knot point at the middle $\left(\xi=\frac{1}{2}\right)$. These new control points satisfy the negativity constraint and then will help to check and better explore the admissible design.

Subdivision operation is a well known flexibility given by B-spline function. The idea was first defined for Bezier curves and surfaces with the Casteljau algorithm; see [61]. This algorithm allows to evaluate a Bezier function at some given parametric coordinates and also, at the same time, to split or subdivise the Bezier curve at that specific parametric coordinate. The subdivision technique has been generalized for B-spline functions by CoxDe-Boor [60], [36].

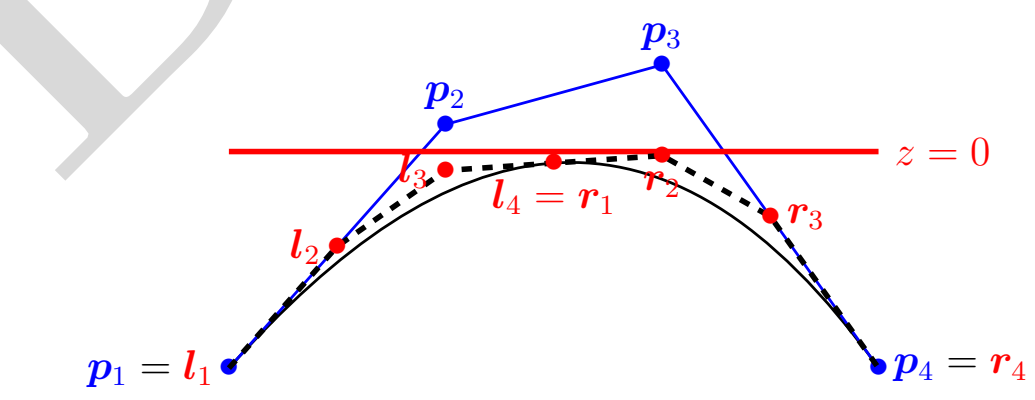

Figure 3: Illustration of subdivision on the B-spline curve $C_{p}$. The new calculated control points $\left\{\boldsymbol{l}_{i}, \boldsymbol{r}_{i}\right\}, i \in\{1, \cdots, 4\}$ satisfy the sufficient conditions 
Hence, one can choose a certain level of subdivision a priori at which the "sufficient constraints" will be checked on the new computed control points and then get a good exploration of the design space.

\section{Formulating the Shape and Anisotropy Optimization Problem}

Thanks to the isogeometric approach the shape and the material properties are driven by some control points. We set subsequently the optimal design problem in the polarisogeometric framework introduced above.

Let us denote by $\boldsymbol{p}_{i}, i \in\left\{1, \cdots, \tilde{n}^{12}\right\}$ the control points associated to the parameterization of the shape, and assume that the shape $\Omega=\Phi(\omega)$ is subjected to:

- some box constraints:

$$
\forall \boldsymbol{\xi} \in \omega, l_{i} \leq \boldsymbol{\Phi}(\boldsymbol{\xi}) \cdot \vec{e}_{i} \leq u_{i}, \forall i \in\{1,2,3\}
$$

where $l_{i}$ and $u_{i}$ are respectively the lower and upper bounds on the $\mathrm{i}^{\text {th }}$ Cartesian coordinate.

These constraints can be taken into account in the same way as the constraints on the elastic tensor, by using Proposition 4.1: the sufficient constraints will be put on the control points of the geometry and read

$$
\boldsymbol{p}_{j} \cdot \vec{e}_{i} \in\left[l_{i}, u_{i}\right], \forall j \in\left\{1, \cdots, \tilde{n}^{12}\right\},
$$

- bounds on the surface area $\mathcal{A}$ of the shell

$$
l_{0} \leq \mathcal{A} \leq u_{0}
$$

where $l_{0}$ and $u_{0}$ are the lower and upper bounds on the area $\mathcal{A}$

$$
\mathcal{A}=\int_{\omega} \sqrt{a} d \xi^{1} d \xi^{2}
$$

- a fix place boundary condition on its edge $\Gamma=\boldsymbol{\Phi}(\gamma)$ of parametric boundary $\gamma \in \partial \bar{\omega}$. The four parametric boundaries are the four edges of the unit square domain, i.e

$$
\gamma=\{0\} \times I, \gamma=\{1\} \times I, \gamma=I \times\{0\} \text { or } \gamma=I \times\{1\} \text { with } I=[0,1] .
$$

Let $I_{\omega}=\left\{1, \cdots, \tilde{n}^{12}\right\}$ denote the set of indexes of the control points of $\Omega=\Phi(\omega) ; I_{\gamma}$ be the subset of indexes of the control points relative to $\Gamma=\boldsymbol{\Phi}(\gamma)$ such that $\left|I_{\gamma}\right|=\tilde{n}^{\alpha}, \alpha \in\{1,2\}$ and $\bar{I}_{\gamma}=I_{\omega} \backslash I_{\gamma}$ the complementary subset of indexes, $n=\left|\bar{I}_{\gamma}\right|=\tilde{n}^{12}-\tilde{n}^{\alpha}$. The space of 
admissible shape $\mathcal{E}_{\Omega}$ is then

$$
\begin{aligned}
\mathcal{E}_{\Omega}= & \left\{\Omega=\boldsymbol{\Phi}(\omega) \text { s.t. } \boldsymbol{\Phi} \in \mathcal{B}_{2}(\boldsymbol{\Sigma}, \boldsymbol{d})\right. \\
& \left.\hat{\boldsymbol{p}}=\left\{\boldsymbol{p}_{i}\right\}_{i \in \bar{I}_{\gamma}} \in \mathbb{R}^{3 \times n} \text { and }(52),(53) \text { hold }\right\} .
\end{aligned}
$$

As well as the polar parameters moduli $R_{0}^{K}$ and $R_{1}$, the orthotropy angle is assumed to be parameterized as a B-spline function of given knot vectors and degrees which can be chosen arbitrarily of the ones of $R_{0}^{K}$ and $R_{1}$.

Let $\hat{\boldsymbol{\Phi}}_{1}=\left\{\Phi_{1}^{i}\right\}_{i=1}^{n_{\Phi}} \in \mathbb{R}^{n_{\Phi}}$ be the corresponding control points. For notation simplicity, let $n_{r}=n^{12}$, and let $\hat{\boldsymbol{r}}_{1}=\left\{r_{1}^{i}\right\}_{i=1}^{n_{r}} \in \mathbb{R}^{n_{r}}$ and $\hat{\boldsymbol{r}}_{0}=\left\{r_{0}^{i}\right\}_{i=1}^{n_{r}} \in \mathbb{R}^{n_{r}}$ be the control points associated to the parameterizations of $R_{1}$ and $R_{0}^{K}$. The design space associated to the elastic tensor is

$$
\mathcal{E}_{\mathbb{E}}=\mathcal{E}_{\mathbb{E}}^{\text {geom }} \text { or } \mathcal{E}_{\mathbb{E}}=\mathcal{E}_{\mathbb{E}}^{\text {elas }},
$$

depending on whether the structure is a homogeneous or a laminated shell. Also, there are two possible settings of the spaces $\mathcal{E}_{\mathbb{E}}^{\text {geom }}$ and $\mathcal{E}_{\mathbb{E}}^{\text {elas }}$ depending on the kind of parameterization used for the polar parameters. For instance, in the conformal case, we have

$$
\begin{aligned}
\mathcal{E}_{\mathbb{E}}^{\text {elas }}= & \left\{\mathbb{E}:=\mathbb{E}\left(\hat{\boldsymbol{\Phi}}_{1}, \hat{\boldsymbol{r}}_{0}, \hat{\boldsymbol{r}}_{1}\right) \text { s.t. } \hat{\boldsymbol{r}}_{\alpha}, \alpha \in\{0,1\},\right. \\
& \text { satisfy (46) and } \left.\hat{\boldsymbol{\Phi}}_{1} \in[-\pi, \pi]^{n_{\Phi}}\right\}
\end{aligned}
$$

and

$$
\begin{aligned}
\mathcal{E}_{\mathbb{E}}^{\text {geom }}= & \left\{\mathbb{E}:=\mathbb{E}\left(\hat{\boldsymbol{\Phi}}_{1}, \hat{\boldsymbol{r}}_{0}, \hat{\boldsymbol{r}}_{1}\right) \text { s.t. } \hat{\boldsymbol{r}}_{\alpha}, \alpha \in\{0,1\},\right. \\
& \text { satisfy (47) and } \left.\hat{\boldsymbol{\Phi}}_{1} \in[-\pi, \pi]^{n_{\Phi}}\right\} .
\end{aligned}
$$

Let us consider the compliance objective function $\hat{j}$

$$
\begin{aligned}
\hat{j}: \mathbb{R}^{n} \times \mathbb{R}^{n_{\Phi}} \times \mathbb{R}^{n_{r}} \times \mathbb{R}^{n_{r}} & \rightarrow \mathbb{R} \\
{\left[\hat{\boldsymbol{p}} ; \hat{\boldsymbol{\Phi}}_{1}, \hat{\boldsymbol{r}}_{0}, \hat{\boldsymbol{r}}_{1}\right] } & \mapsto \hat{j}\left(\hat{\boldsymbol{p}} ; \hat{\boldsymbol{\Phi}}_{1}, \hat{\boldsymbol{r}}_{0}, \hat{\boldsymbol{r}}_{1}\right)=\frac{1}{2} a([\boldsymbol{u}, \boldsymbol{s}],[\boldsymbol{u}, \boldsymbol{s}]) .
\end{aligned}
$$

The optimal design problem is: Find the optimal solution $\left[\Phi^{*}, \mathbb{E}^{*}\right]$ such that

$$
\left[\Phi^{*} ; \mathbb{E}^{*}\right]=\arg \min \hat{j}\left(\hat{\boldsymbol{p}} ; \hat{\boldsymbol{\Phi}}_{1}, \hat{\boldsymbol{r}}_{0}, \hat{\boldsymbol{r}}_{1}\right) \text { s.t. }[\Phi, \mathbb{E}] \in \mathcal{E} .
$$

After a brief description of the practical formulation and steps of the optimal design problem in the framework of the polar-isogeometric approach, we give in the next Section some numerical examples.

\section{Some examples}

For the numerical resolution of the examples considered in this Section, we have used NLOPT, a free/open-source library for NonLinear OPTimization. It includes the implementation of numerous optimization algorithms adapted for global and local optimiza- 
tions. The library involves different types of algorithm such as, among others, Moving Asymptote Method (MMA) or COBYLA (Constrained Optimization by Linear Approximation), which can be gradient-based or derivative-free. We have used the COBYLA algorithm which appears to yield the best optimization results among the different algorithms.

In all the examples, we always start from a shell in the form of a flat domain with a given uniform distribution of the anisotropic parameters (e.g., an isotropic distribution). Hence, we begin all the iterations starting from a plate; during the computation, the plate is more and more transformed into a shell and at the same time anisotropy changes pointwise. During this process, the elastic energy, at the beginning entirely stored as bending energy in the plate, transforms continuously more and more to membrane energy. The ideal situation, corresponding to the optimal shell, is the one where all the strain energy of the shell is in the form of membrane energy, i.e. when the bending energy of the shell vanishes everywhere.

This is the process driving the initial plate to the final stiffest shell, acting simultaneously upon shape, i.e. geometry, and anisotropy, i.e. elasticity. So, this process can help to investigate the mutual influence of these two aspects on the morphogenesis of optimal anisotropic shells.

Material properties: In the forthcoming examples, the elastic coefficients of the basic material (i.e. of the basic layer, for the case of a laminate) are:

$$
\begin{aligned}
E_{2} & =E=9000 \times 10^{6} \mathrm{~Pa} \\
E_{1} & =E_{3}=161 \times 10^{6} \mathrm{~Pa} \\
\nu_{12} & =\nu_{23}=\nu=0.26 \\
\nu_{13} & =0.26 \text { and } G_{13}=\frac{E}{1+\nu} \\
G_{12} & =G_{23}=61 \times 10^{6} \mathrm{~Pa}
\end{aligned}
$$

The polar parameters corresponding to the plane reduced elastic tensor are:

$$
\begin{aligned}
& T_{0}=1.17 \times 10^{9} \mathrm{~Pa}, T_{1}=1.16 \times 10^{9} \mathrm{~Pa} \\
& R_{0}^{K}=1.11 \times 10^{9} \mathrm{~Pa}, R_{1}=1.11 \times 10^{9} \mathrm{~Pa}, \text { and } \Phi_{1}=0
\end{aligned}
$$

We consider up to three different types of optimal design problems, in order to analyze the incidence of the anisotropy on the shape:

- optimal shape design with an isotropic material (by setting $R_{0}^{K}=R_{1}=0$ );

- optimal shape design with the specified anisotropic material and a fixed material orientation throughout the shell;

- joint optimal design of the shape and anisotropy, included the material orientation.

In all the cases, geometric bounds (39) are used in the calculations. The shape is parameterized by cubic B-spline polynomial of clamped knot vectors and 4 control points in each coordinate direction. The polar parameters are defined through B-spline polynomial of 
degrees $d$ of clamped knot vectors of the form

$$
\Sigma^{\alpha}=\{\underbrace{0, \cdots, 0}_{d+1}, \underbrace{1, \cdots, 1}_{d+1}\}, \alpha \in\{1,2\} .
$$

The geometry is subjected to box constraints on the control points and to a bounded area constraint of the form

$$
l_{0}=\left(1-\epsilon_{\text {tol }}\right) \mathcal{A}_{0} \leq \mathcal{A} \leq u_{0}=\left(1+\epsilon_{\text {tol }}\right) \mathcal{A}_{0}
$$

that is, the relative variation of the design area with respect to the initial shell area $\mathcal{A}_{0}$ is $\epsilon_{t o l}$.

In order to tailor efficiently the locally variable elastic properties, it can be relevant to use an assembling of patches for the parameterization of the polar parameters. This does not present any additional difficulty since, provided that the material frame of two adjacent patches are consistent, the continuity on the polar parameters (if necessary) is easily obtained by equating the control parameters at the interfaces of the adjacent patches.

\subsection{Optimal Design of a Circular Dome}

The first case concerns the design of a circular dome submitted to its own weight and simply supported at its boundary. The geometry of the initial structure, a circular plate, and the problem data are represented in figure 4 and table 1. For symmetry reasons, the optimization is performed on a quarter of the structure and symmetry conditions are imposed on the elastic displacement.

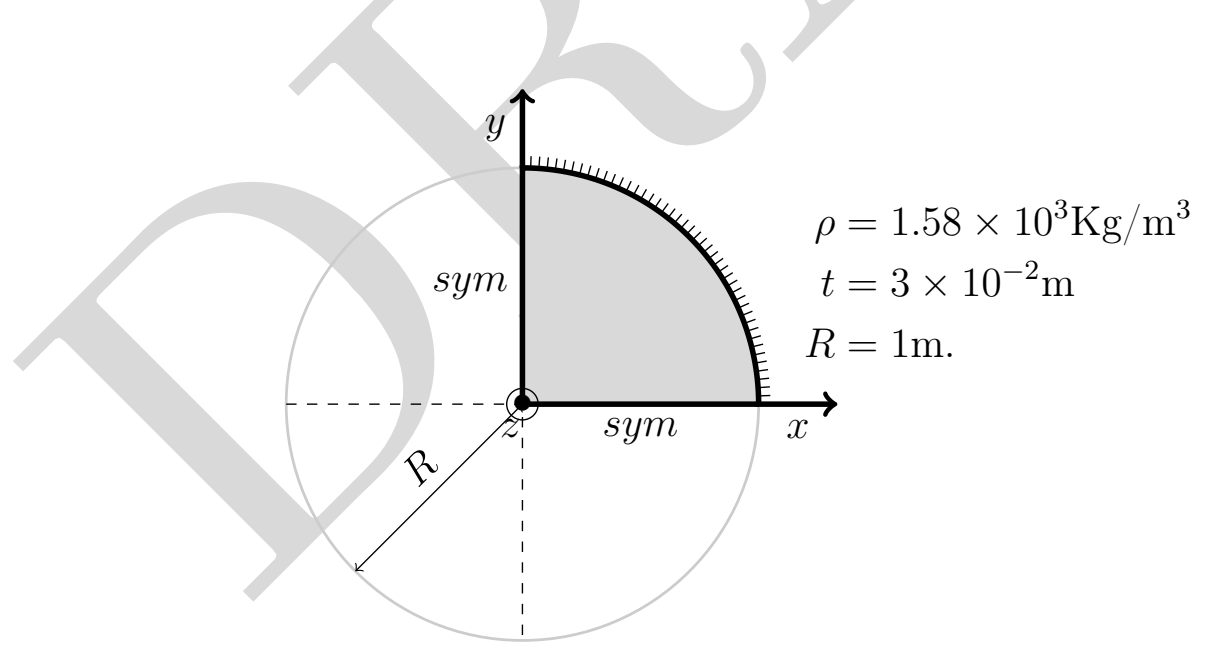

Figure 4: Circular plate geometry and boundary conditions.

First case: Shape design with fixed isotropic material

The optimal shape found for the shell is represented in Figure 5. We have checked that 
Table 1: Design problem relative to the circular plate

\begin{tabular}{|c|c|}
\hline $\begin{array}{l}\text { Geometry \& } \\
\text { loading: }\end{array}$ & $\begin{array}{l}\text { Circular plate of radius } R=1 \mathrm{~m} \\
\text { subjected to its own weight }\end{array}$ \\
\hline \multirow[t]{3}{*}{ Constraints: } & $\begin{array}{l}\text { - Symmetries with } C^{1} \text { regularity throughout the planes of } \\
\text { symmetry } x-z \text { and } y-z \\
\text { - Fix place constraint on the simply supported boundary } \\
\text { - Box constraints } \\
\text { - Bounded area constraints } \epsilon_{t o l}=0.3\end{array}$ \\
\hline & Anisotropy \\
\hline & $R_{0}^{K}, R_{1}$ \\
\hline Knots & $\Sigma^{\alpha}=\{0,0,0,0,1,1,1,1\}$ \\
\hline \multirow[t]{2}{*}{ Constraints } & - geometric bounds (47) bounds on the angle - see (58) \\
\hline & Anisotropy \\
\hline $\begin{array}{l}\text { Number of design } \\
\text { variables }\end{array}$ & 12 \\
\hline
\end{tabular}

the optimal structure is a shell whose meridional profile is a catenary. It is well known that the catenary is the form of equilibrium of an arch of constant thickness under the action of its own weight in which all the internal actions reduce to a pure normal force (this result is due to R. Hooke, [62], see also [63] or [64]). Such an arch shape optimizes also its stiffness, because all the strain energy is stored in the structure under the form of extension energy, while the bending one is reduced to zero; this condition, as well known, corresponds to the maximum stiffness.

The optimal shell so obtained is, in the same way, submitted to only membrane internal actions, so that the bending energy vanishes. Actually, the membrane energy part for the optimal solution is $E_{m}=98.35 \%$. Figure 6 shows the evolution of the compliance, i.e., of the strain energy, and the contributions of the membrane and bending parts to the strain energy, along the optimization procedure. We can remark the migration of the strain energy from the dominant initial form of bending energy to the final prevailing form of membrane energy.

Second case: anisotropy and shape design The second case that we consider for this example is the joint optimization of the polar parameters and of the shape. The polar parameters are subjected to geometric bounds on their control points and are defined as B-spline of degrees $d=3$. The optimal shape and the orthotropy direction are plotted in Figure 7. Also in this case the optimal shape is that of a shell with meridional sections in the form of a catenary. The polar parameters moduli are plotted in Figure 8. One remarks that these parameters are not only uniform throughout the structure, but also 


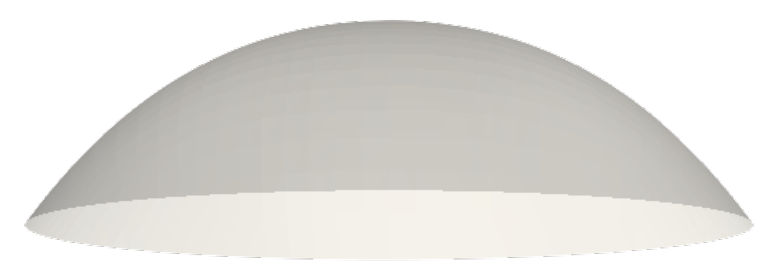

Figure 5: Example 1: optimal shape for isotropic material.
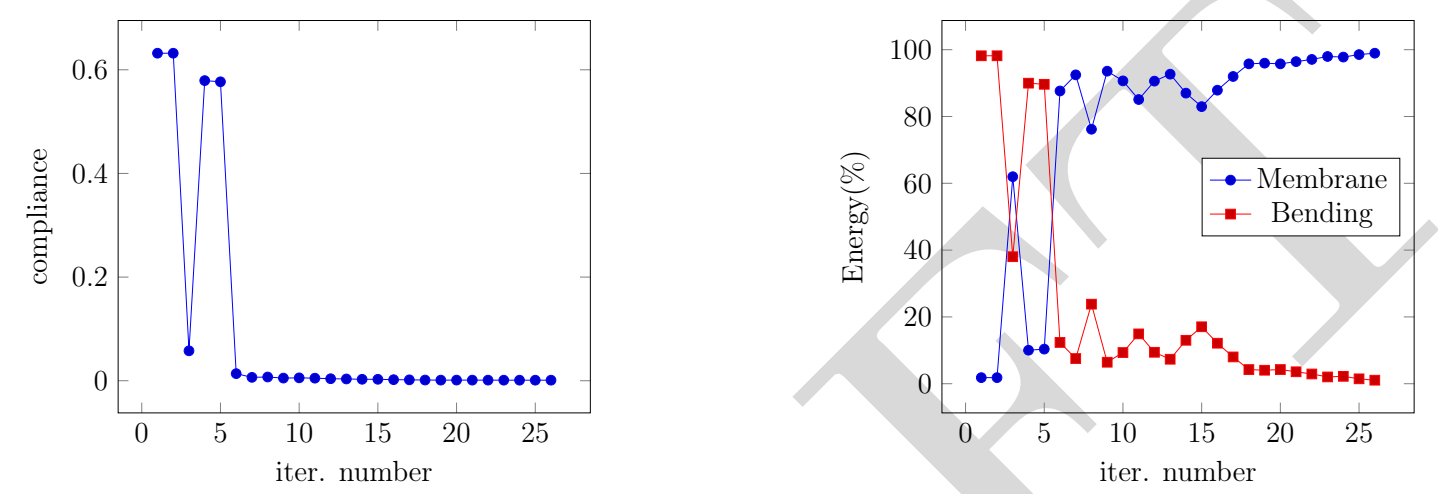

Figure 6: Example one, shape optimization with isotropic material: evolution of the compliance through iterations (left) and ratio of the membrane and bending energy to the total strain energy (right).

that both of them take the highest possible value: the optimal shell is that with the highest possible degree of anisotropy of the two anisotropic phases. We also remark that the only orthotropy type is the one with $K=0$.

Figure 9 shows the variation of the compliance and once again the migration of the strain energy from the bending to the membrane form throughout the optimization: for the optimal design, the membrane part is $93.34 \%$ of the total strain energy.

Table 2: Summary of the global results concerning the first example.

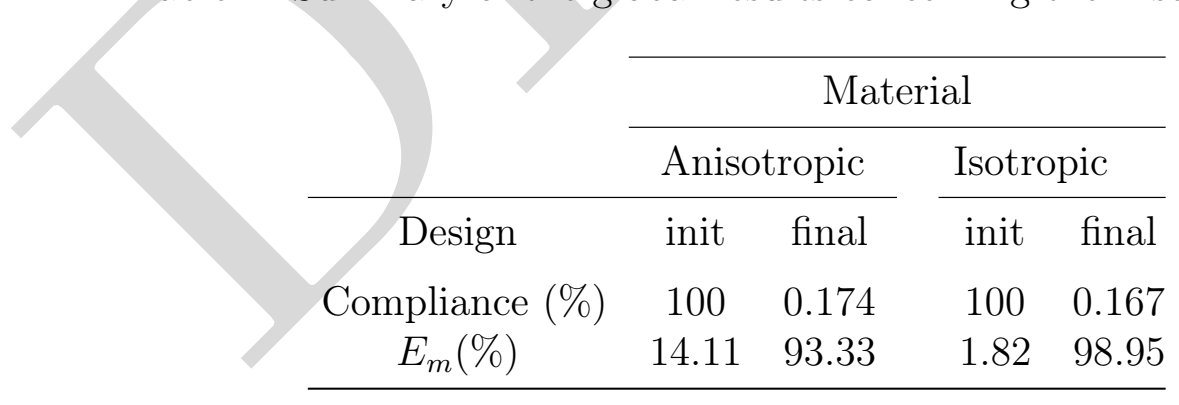

\subsection{Optimization of a Conical Shell}

As second example, we consider the optimization of a conical shell: the starting structure is a holed circular plate which is simply supported at its external boundary and subjected to an uniformly distributed vertical load at its inner circular boundary. The radius of the 


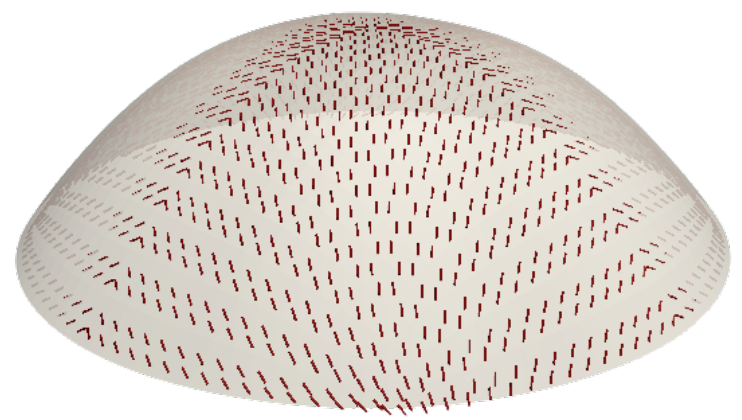

Figure 7: Example one, shape and material optimization: optimal shape and orthotropy direction field.
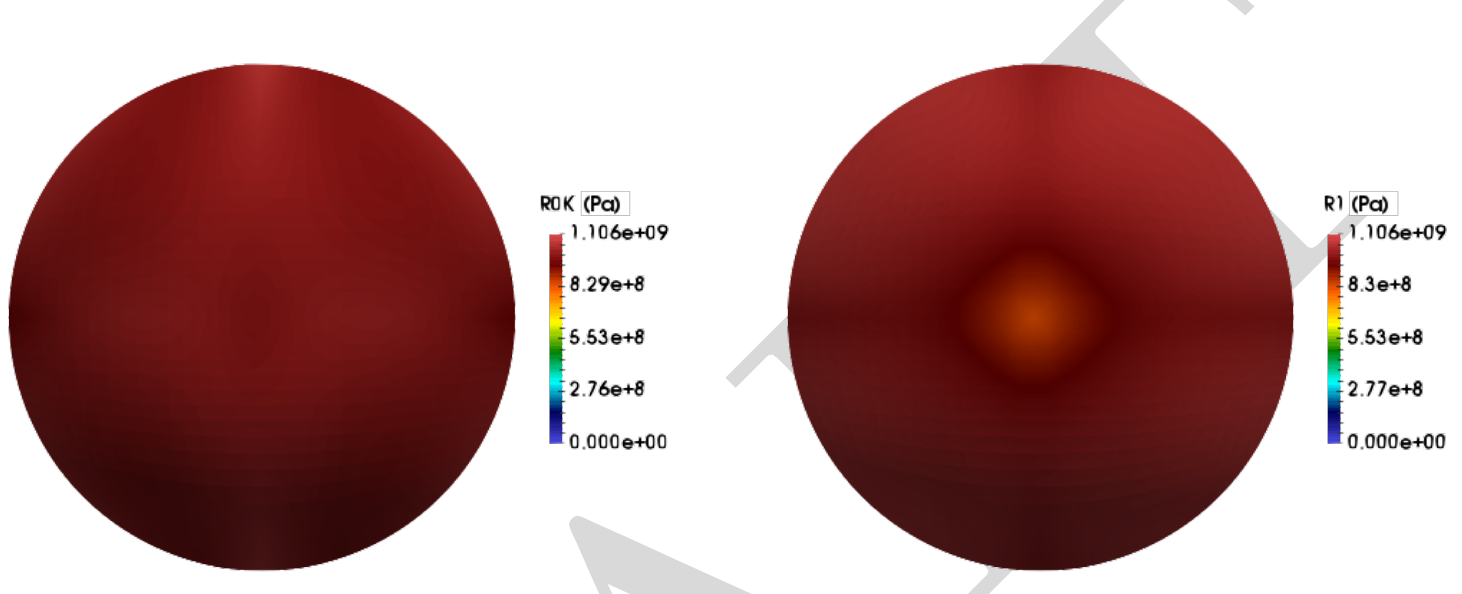

Figure 8: Example one, shape and anisotropy optimization: optimal polar moduli fields.

circular plate is $R=0.8 \mathrm{~m}$ while that of the hole is $r=\frac{R}{3}$. The geometry and conditions are shown in Figure 10 and Table 3.

For symmetry reasons, the optimization is performed on a quarter of the structure. The geometry is defined by two sub-structures joined together as described in Figure 10 and the optimization problem set up is reported in Table 3. The control points associated to the circular boundary with simply-supported condition are kept fixed while those defining the internal crown, which carries the applied load, are constrained to have the same $z$ coordinate, i.e., the internal circular boundary can move rigidly.

\section{First case: shape design with fixed isotropic material}

The optimal shape found is plotted in Figure 11, while the variation of the compliance and of membrane and bending energy parts during the optimization procedure are plotted in Figure 12.

Also in this case we can remark the migration of the strain energy from the bending to the membrane form: at the end of the calculation, this last is $98.88 \%$ of the whole strain energy.

For this example, the optimal shape is a conical surface, to which corresponds a strain 

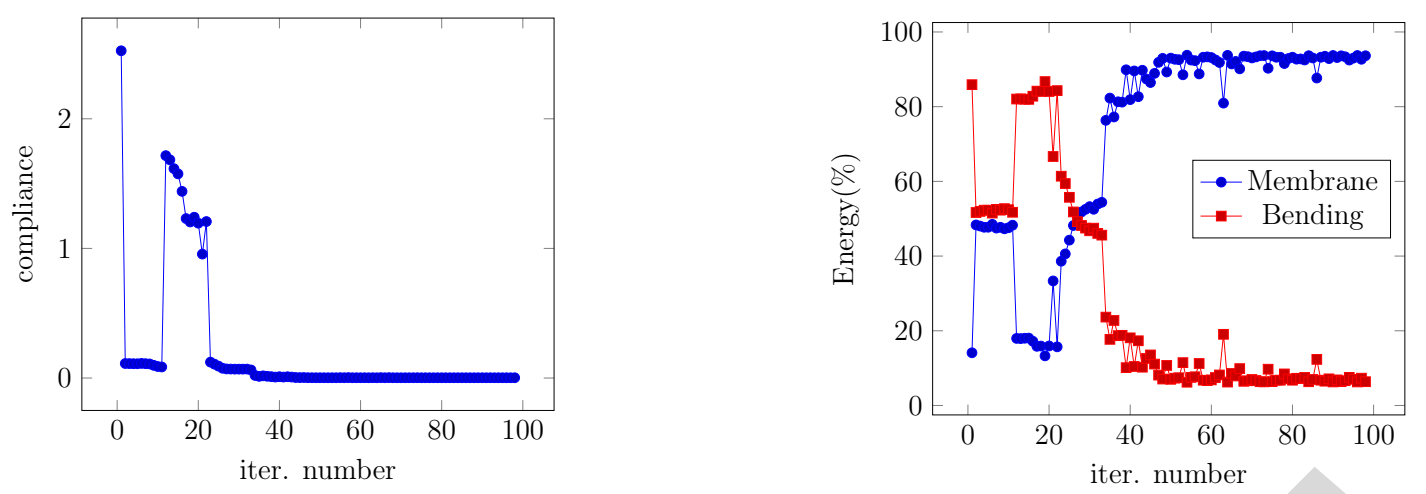

Figure 9: Example one, shape and material optimization: evolution of the compliance through iterations (left) and ratio of the membrane and bending energy to the total strain energy (right).

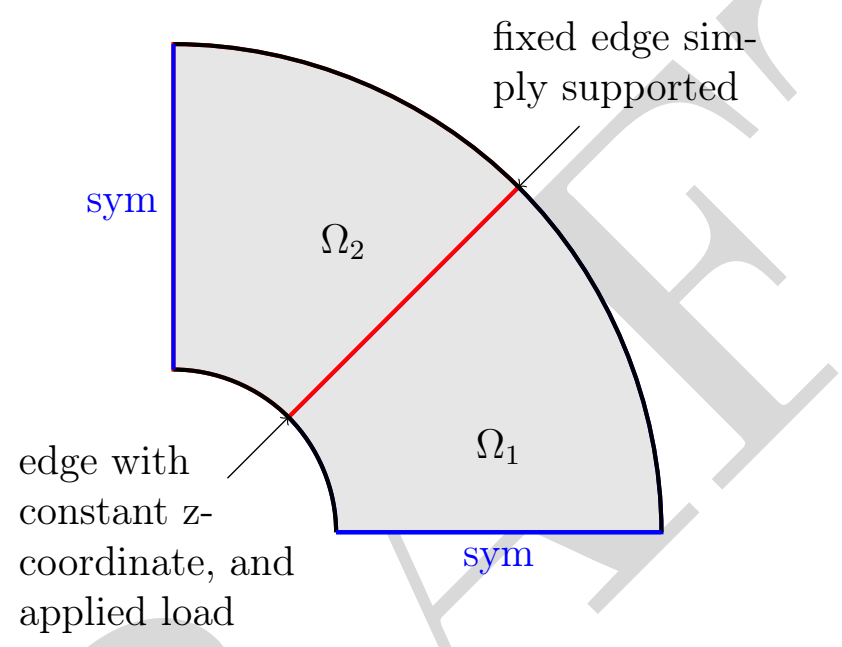

Figure 10: Example two: geometry and boundary conditions.

energy completely stored under the form of membrane energy. The result shown in Figure 11 is not exactly a conical surface, but it is close to it. This is due to the fact that the solution has not yet perfectly converged, which is attested by the fact that the membrane energy is not $100 \%$ of the whole elastic energy stored in the shell.

\section{Second case: shape design with fixed anisotropic material.}

We consider now the optimization of the shape made with the anisotropic material specified in Sect. 6 under the same constraints on the geometry as in the previous case. In addition, we fix the angle of orthotropy: $\Phi_{1}=\frac{\pi}{4}$. The optimal shape so found is shown in Figure 13. In this case we do not obtain a shell with circular cross section, but a wrinkled surface, which is the apparent consequence of using a fixed anisotropic material with a fixed orientation throughout the shell. Unlike in the previous case, however, the wrinkles have an almost rectilinear profile, closer to a conical shape than before. We remark also that, as well known, wrinkled membranes are very stiff structures and it is interesting to notice that when anisotropy enters the design, the optimal shape becomes a wrinkled surface. Figure 14 shows the variation of the strain energy and of the membrane and bending parts throughout optimization. In this case, though the tendency is the same of 
Table 3: Description of the conical shell design problem.

\begin{tabular}{|c|c|}
\hline $\begin{array}{l}\text { Geometry \& } \\
\text { loading: }\end{array}$ & $\begin{array}{l}\text { Holed circular plate of outer radius } R=0.8 \mathrm{~m} \text { and inner } r=\frac{R}{3} \\
\text { Loaded at its inner boundary }\end{array}$ \\
\hline \multirow[t]{3}{*}{ Constraints: } & $\begin{array}{l}\text { - Symmetries with } C^{1} \text { regularity } \\
\text { throughout the planes of symmetry } \\
\text { - Fix place constraint on the simply supported boundary } \\
\text { - The inner loaded circle remains a circle } \\
\text { - Box constraints } \\
\text { - Bounded area constraints } \epsilon_{t o l}=\frac{3}{2}\end{array}$ \\
\hline & Anisotropy \\
\hline & $R_{0}^{K}, R_{1}$ \\
\hline Knots & $\Sigma^{\alpha}=\{0,0,0,1,1,1\}$ \\
\hline \multirow[t]{2}{*}{ Constraints } & - geometric bounds (47) bounds on the angle - see (58) \\
\hline & Anisotropy \\
\hline $\begin{array}{l}\text { Number of design } \\
\text { variables }\end{array}$ & 25 \\
\hline
\end{tabular}

the previous cases, the final membrane energy is only $87.3 \%$ of the whole strain energy stored in the shell; the remaining $12.7 \%$ is in the form of bending energy, which is due to the presence of the wrinkles.

\section{Third case: shape and anisotropy optimal design}

We presently consider the joint optimization of the shape and material properties for this second example. The parameterization considered for the polar parameters corresponds to $d=2$, thus, considering also the continuity condition between the two patches, there are 15 design variables for each polar parameter. Figure 15 shows the optimal shape and orthotropy direction.

We remark that the optimal shape is, like in the first case, a shell close to a conical surface; in particular, in this case the final surface is closer to a conical one than in the first case. Also, we notice that the optimal orientation is the same everywhere: the highest elastic modulus is in the meridional direction, which seems a logical result. Unlike the optimal material orientation, which is constant throughout the shell, the optimal distribution of the polar anisotropic moduli $R_{0}^{K}$ and $R_{1}$ is not constant, see Figure 16 . 


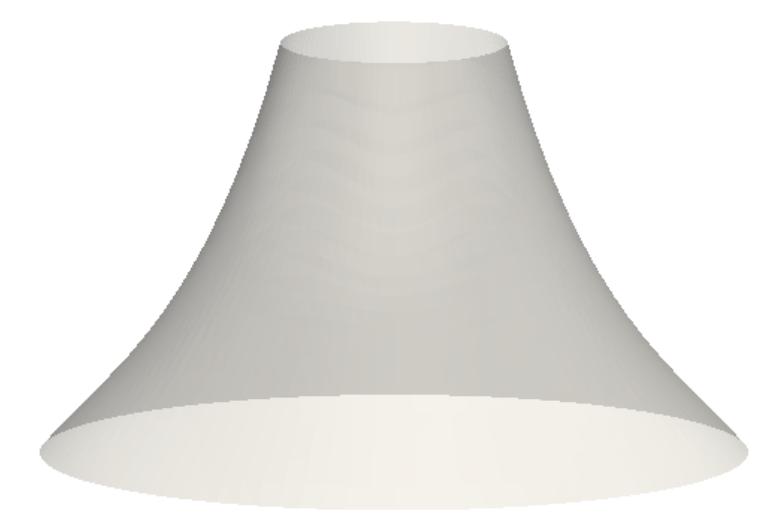

Figure 11: Example two: optimal shape for the isotropic case.
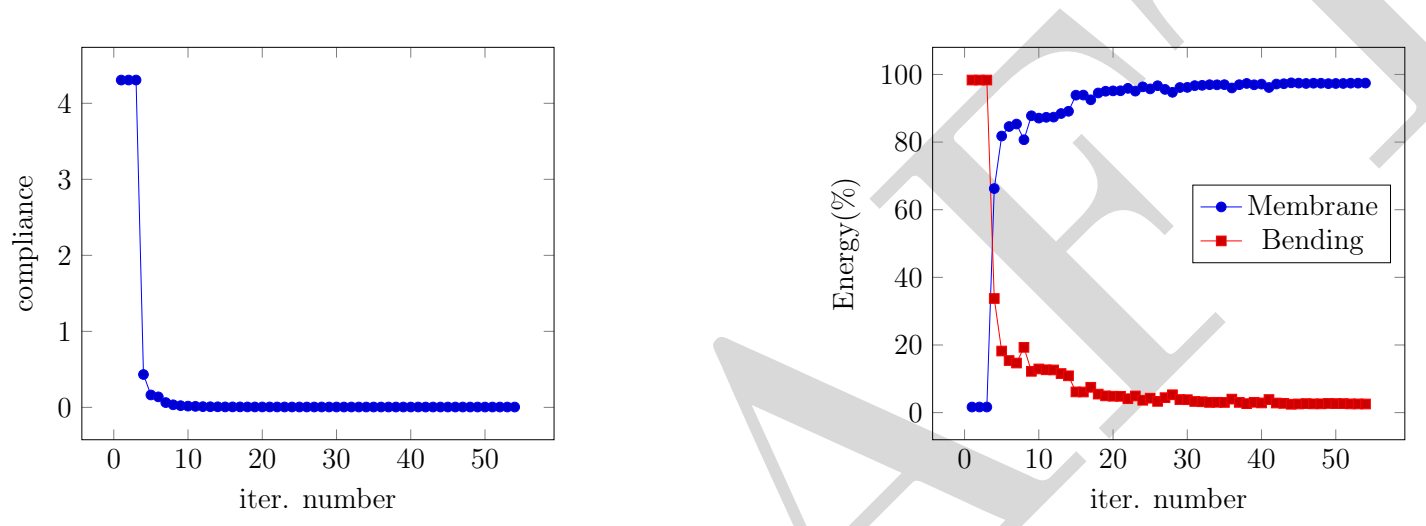

Figure 12: Example two, shape optimization with isotropic material: evolution of the compliance through iterations (left) and ratio of the membrane and bending energy to the total strain energy (right).

In particular, the field of the parameter $R_{0}^{K}$ is uniform and almost constant over all the shell with the presence of only one kind of orthotropy $K=0$, while $R_{1}$ changes, in particular it increases from top to bottom.

We remark also that the optimal material orientation is different from that, fixed a priori, of the previous case. The consequence of this is the different optimal shape of the shell, that now is not wrinkled.

Figure 17 shows also for this case the variation along iterations of the strain energy and of its membrane and bending parts. The variation is analogous to that of the previous cases; in particular, one can notice that the convergence is practically reached after 60 iterations and at the end the membrane energy amounts to $97.2 \%$ of the whole elastic energy stored in the shell.

The overall results for the three cases considered for this second example are shown in Table 4. We can remark that the most effective case is the third one, with a final compliance which is just the $0.004 \%$ of the initial one. This shows that acting simultaneously on the geometry and on the material distribution is advantageous. 


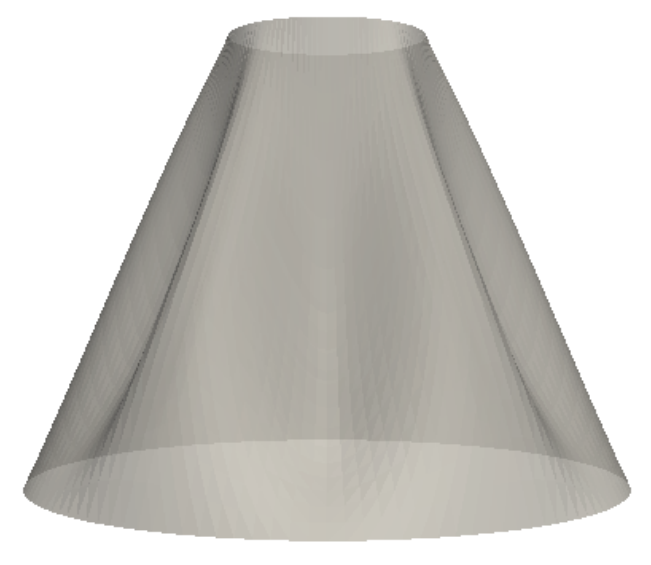

Figure 13: Example two: optimal shape for fixed anisotropic material.
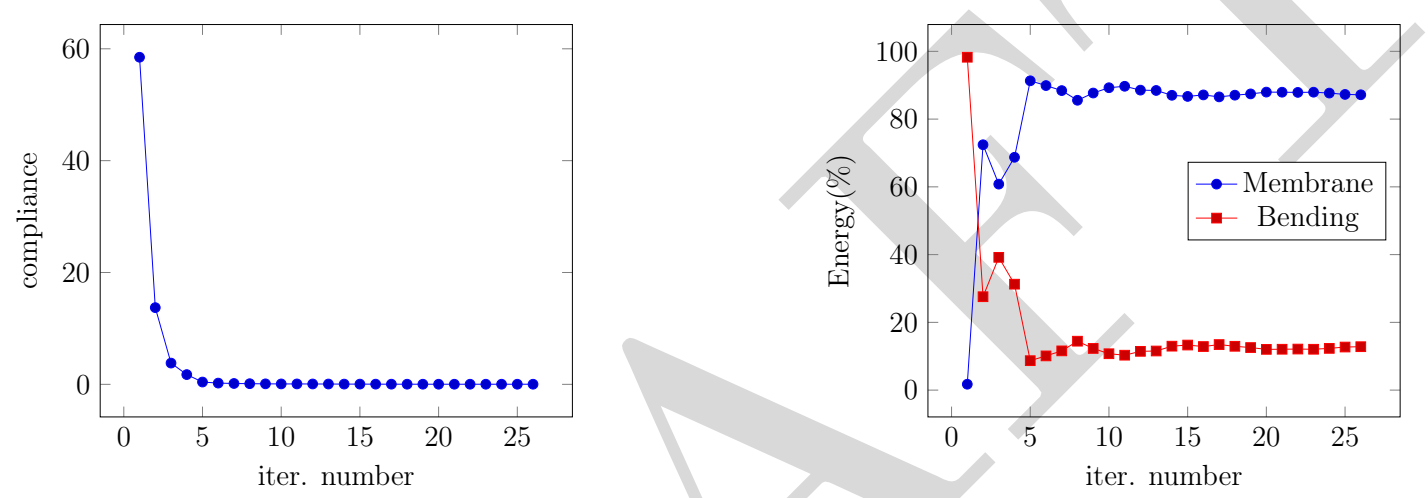

Figure 14: Example two: optimal shape and material orientation for fixed anisotropic material. Evolution of the compliance through iterations (left) and ratio of the membrane and bending energy to the total strain energy (right).

\subsection{Plate Submitted to a Torsional Load}

The last example that we consider is a square plate with a side of $1 \mathrm{~m}$, clamped at one side and subjected to two equal but opposite loads applied at the free corners. On the whole, the plate is hence submitted to a torsional action. The geometry of the plate is sketched in Figure 18, while in Table 5 we show the data of the problem. The two concentrated loads producing the torsion of the plate have a value $f=1000 \mathrm{~N}$. The plate is defined by an assembling of $2 \times 2$ square plates of length $0.5 \mathrm{~m}$, each being parameterized through a cubic B-splines with open knot vectors and four control points in each parametric direction. Finally a $C^{1}$-regularity is imposed at the junction of the patches. The design is constrained to preserve the boundary: the final shape of the optimal shell must have the same boundary of the original square plate. The orthotropy is initially oriented with an angle $\Phi_{1}=0$ with respect to the $x$-axis direction.

\section{First case: shape design with fixed isotropic material}

As usual, we first consider that the plate is made with the isotropic material obtained putting $R_{0}^{K}=R_{1}=0$. Figure 19 shows, on the left, the optimal shape so found. It is interesting to notice that this shape corresponds with one of the fundamental vibration 


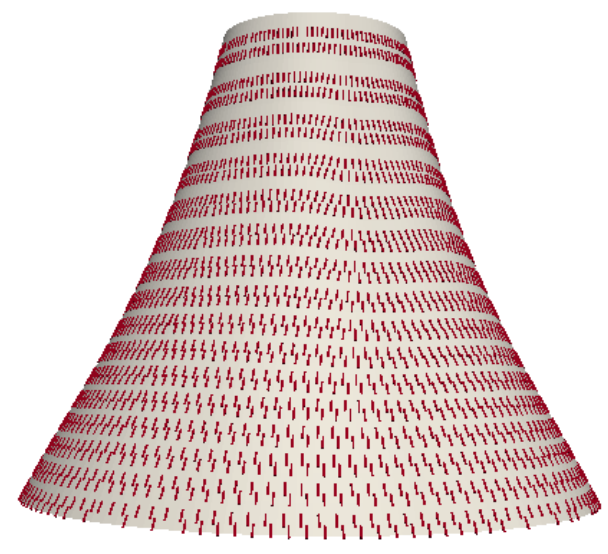

Figure 15: Example two, shape and anisotropy optimization: optimal shape and material orientation.
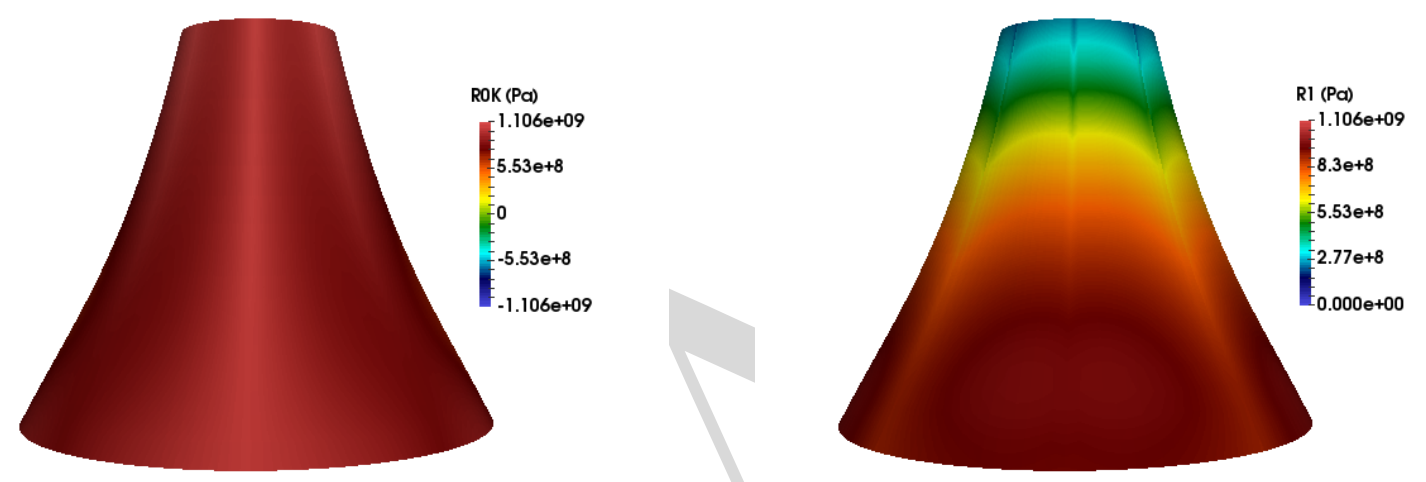

Figure 16: Example two, shape and anisotropy optimization. Distribution of the optimal polar moduli $R_{0}^{K}$ and $R_{1}$.

modes of the plate when it is simply supported along all its boundary.

\section{Second case: shape design with fixed anisotropic material}

Like for the previous example, we consider now the optimal shape design with the anisotropic material given in Sect. 6 and with the material orientation that is fixed everywhere to $\Phi_{1}=0$. The final optimal shape is shown in Figure 19, on the right. Now, the optimal shape has remarkably changed with respect to the previous case: the waves are more numerous and they decrease going towards the clamped edge. It is interesting to remark that the greatest curvatures in the shell shape are located near the loaded corners.

\section{Third case: shape and anisotropy optimal design}

Finally, we consider the joint shape and anisotropy optimal design. Figure 20 shows the optimal shape and material orientation. The optimal shape changes again with respect to the two previous cases; the optimal distributions of the polar moduli $R_{0}^{K}$ and $R_{1}$ are 

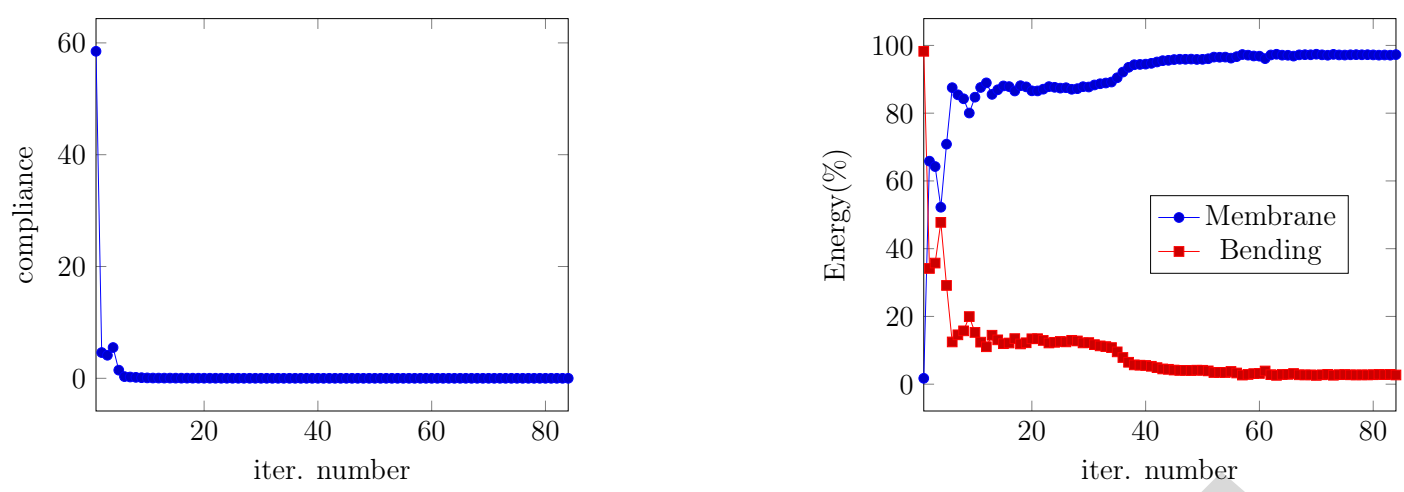

Figure 17: Example two: shape and material optimization. Evolution of the compliance through iterations (left) and ratio of the membrane and bending energy to the total strain energy (right).

Table 4: Summary of the different results concerning the second example (final 1: final value for the first case, final 2 for the second one and final 3 for the third one).

\begin{tabular}{|c|c|c|c|c|}
\hline & \multirow{2}{*}{\multicolumn{4}{|c|}{ Material }} \\
\hline & & & & \\
\hline & \multicolumn{3}{|c|}{ Anisotropic } & Isotropic \\
\hline Design & init & final 2 & final 3 & init final 1 \\
\hline Compliance (\%) & 100 & 0.036 & 0.004 & $100 \bigcirc 0.105$ \\
\hline$E_{m}(\%)$ & 1.74 & 87.3 & 97.2 & $1.65 \quad 98.9$ \\
\hline
\end{tabular}

represented in Figure 21. Like in the previous examples, they are practically constant throughout the structure and equal to their maximal allowed value. Also in this case, there is a unique final type of orthotropy, that with $K=0$.

Table 6 summarizes the different optimization results for this third example. Like in the previous example, also in this case the best result is found for the third case, i.e., when shape and anisotropy are optimized. The optimal shapes so found for this example are rather unexpected and surprising is their similarity with the vibration modes of a simply supported plate. To this purpose, it is interesting to remark how the presence of anisotropy modifies the shape, changing the number of waves in the shell shape.

\section{Conclusions}

In this paper we have proposed a problem in a new field of structural optimization: the joint optimization of shape and material distribution for a shell-like structure. We have also proposed to tackle such a kind of problem by an approach that we have called polar-isogeometric, because it marries two distinct mathematical techniques: the polar formalism for the representation of plane anisotropy and an isogeometric-like approach for the parametrization of both the shell shape and the fields of the polar parameters.

Starting from a flat shape, i.e. from a plate, we have shown through different examples that the proposed method is able to drive the computation towards the optimal solution, the one where the elastic energy of the structure tends to be stored as membrane 


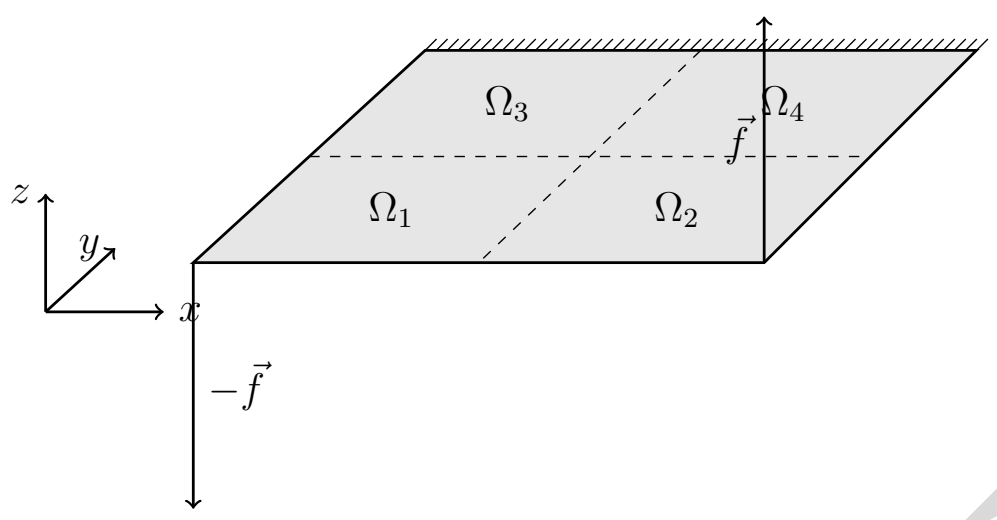

Figure 18: Example three: geometry, actions and boundary conditions.
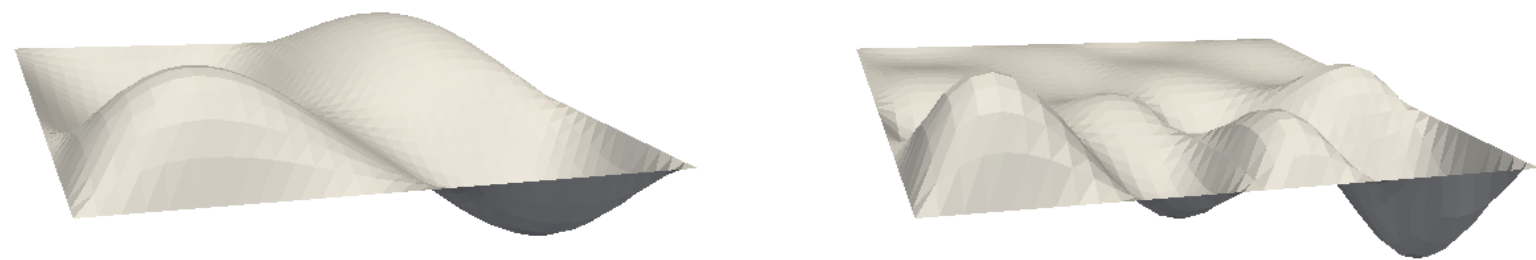

Figure 19: Example three: optimal shapes corresponding to the first and second case (from the left).

energy.

The use of the isogeometric approach, which is almost classical in shape design, has been used also for the parametrization of the variables describing the anisotropy of the shell. This has been done in order to reduce the number of design variables that become, in this approach, the control points of the parameterizations. Using the properties of the spline functions, we have also given a sufficient condition for ensuring the satisfaction of the bounds on the elastic parameters, to be fulfilled everywhere in the shell.

This is just one of the first works in this field and of course different improvements can be imagined, we discuss some of them. As a first point, we indicate the possibility of describing complex shapes using more than one patch. This problem has already been tackled and partially solved in [25]; the matter is delicate, because continuity conditions must be specified for the shape and for the elastic parameter fields. If for the first case, the shape, such conditions are delicate to be written, but rather well defined, for the second one, the elastic parameters, the definition of such continuity conditions among the patches is questionable, different possibilities can be imagined, driving towards different optimal problems.

A second point is the design of laminated shells. This point is rather well solved for plates and it just needs few amendments to the proposed approach: besides taking into account for the geometric instead of the elastic bounds, we need to use a specific procedure for finding a laminate able to have all the properties of the optimal shell, i.e., orthotropic, quasi-homogeneous and with the optimal distribution of $\mathbb{E}$ everywhere. Such a problem has been already solved satisfactorily for different problems concerning plates, see e.g. $[12,14,15,47]$, using metaheuristics, e.g. a genetic algorithm. However, a step further in this direction should be the introduction in the proposed optimization process of some 
Table 5: Example three: data of the optimum problem.

\begin{tabular}{|c|c|}
\hline $\begin{array}{l}\text { Geometry \& } \\
\text { loading: }\end{array}$ & $\begin{array}{l}\text { Square plate of unit length } \\
\text { Torsion applied through two concentrated loads }\end{array}$ \\
\hline \multirow[t]{3}{*}{ Constraints: } & $\begin{array}{l}\text { - } C^{1} \text { regularity constraints between the patches } \\
\text { - Fix place constraint on the boundary } \\
\text { - Box constraints } \\
\text { - Bounded area constraints } \epsilon_{t o l}=0.2\end{array}$ \\
\hline & Anisotropy \\
\hline & $R_{0}^{K}, R_{1}$ \\
\hline Knot & $\Sigma^{\alpha}=\{0,0,0,0,1,1,1,1\}$ \\
\hline \multirow[t]{2}{*}{ Constraints } & - geometric bounds (47) bounds on the angle - see (58) \\
\hline & Anisotropy \\
\hline $\begin{array}{l}\text { Number of design } \\
\text { variables }\end{array}$ & 36 \\
\hline
\end{tabular}

Table 6: Summary of the different results concerning the third example (final 1: final value for the first case, final 2 for the second one and final 3 for the third one).

\begin{tabular}{|c|c|c|c|c|c|}
\hline & & & \multicolumn{3}{|c|}{ Material } \\
\hline & \multicolumn{3}{|c|}{ Anisotropic } & \multicolumn{2}{|c|}{ Isotropic } \\
\hline & init & final 2 & final 3 & init & final 1 \\
\hline Compliance (\%) & 100 & 1.000 & 0.256 & 100 & 5.150 \\
\hline$E_{m}(\%)$ & 3.28 & 91.71 & 94.98 & 16.48 & 93.05 \\
\hline
\end{tabular}

technological constraints, e.g. on the trajectories and densities of the reinforcing fibers, using, for instance, the technique already proposed in [27]. For the case of laminated shells, the use of quasi-homogeneous laminates is almost essential, because the structure is, at least in some parts, submitted to extension and bending. To avoid this assumption means, on one hand, to introduce separately the polar parameters for extension and bending, which doubles the number of design variables for the elastic part, and, on the other hand, to use some approximation of the feasible domain, because the exact bounds of the polar parameters for extension and bending, that are not independent, are still unknown, see [40].

The proposed approach can be used also for other objective functions rather than compliance. In particular, for buckling loads and vibration frequencies, i.e. for problems concerning eigenvalues, the proposed method can be applied almost directly; more difficult is the case of strength, because this is represented by a local functional; a possible 


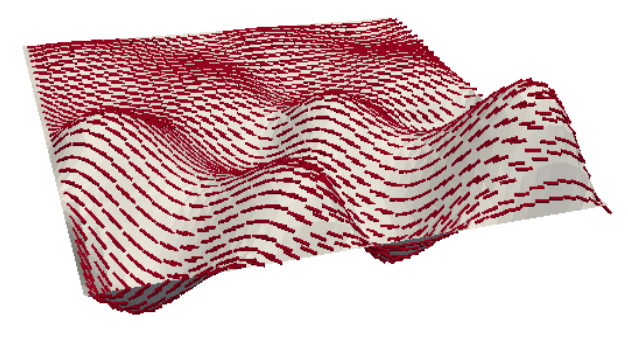

Figure 20: Example three, shape and material optimization: optimal shape and material orientation.

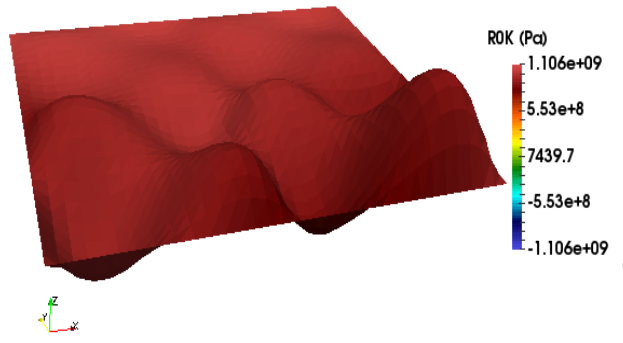

(a) Optimal distribution of $R_{0}^{K}$

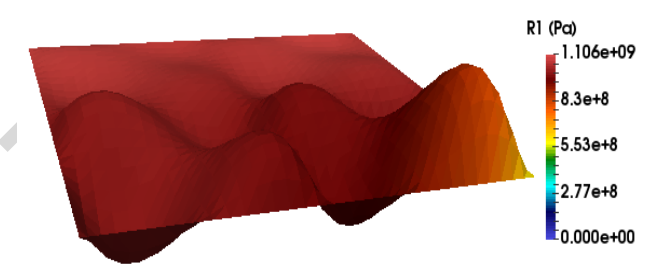

$\operatorname{Lit}_{\rightarrow}^{2}$

(b) Optimal distribution of $R_{1}$

Figure 21: Example three, shape and anisotropy optimization: optimal distribution of the anisotropy polar parameters.

way is that introduced in [12], because the use of the isogeometric approach used in this paper can be easily adapted to the polar parameters describing strength in the case of a tensorial strength criterion.

Finally, the problem considered in this paper has been suggested by industrial applications: automotive, aeronautics, space and sport engineering are more and more interested in the optimal design of composite structures, most of them being in the form of shells. Thanks to the new technologies of fiber placement, the possibility of tailoring anisotropy is today a true reality; so, the goal is to dispose of mathematical methods able to drive the design towards optimal solutions.

However, to our opinion, this approach has a wider interest and significance; in fact, as already said in the text, this approach allows to investigate the reciprocal influence of material properties and geometrical shape in the morphogenesis of 2D space structures; we think, namely, to natural forms, like leafs or some skeletal organisms. This approach could be of interest in trying to investigate how Nature acts when it can dispose of geometry and material.

Acknowledgements: The authors sincerely acknowledge RENAULT SA for its support to this research through the granting of the PhD thesis of D. F. Kpadonou. 


\section{References}

[1] Banichuk, N.V.: Problems and Methods of Optimal Structural Design. Springer US (1983). DOI 10.1007/978-1-4613-3676-1 3

[2] Allaire, G.: Shape Optimization by the Homogenization Method. Springer New York (2002). DOI 10.1007/978-1-4684-9286-6 3

[3] Bendsøe, M.P., Sigmund, O.: Topology Optimization. Springer Berlin Heidelberg (2004). DOI 10.1007/978-3-662-05086-6 3

[4] Gurdal, Z., Haftka, R.T., Hajela, P.: Design and Optimization of Laminated Composite Materials. Wileys, New York (1999) 3

[5] Vannucci, P.: Anisotropic Elasticity. Springer Singapore (2018). DOI 10.1007/ 978-981-10-5439-6 3, 11, 14

[6] Montemurro, M., Vincenti, A., Vannucci, P.: Design of the elastic properties of laminates with a minimum number of plies. Mechanics of Composite Materials 48(4), 369-390 (2012). DOI 10.1007/s11029-012-9284-4 3, 11

[7] Vannucci, P.: Designing the elastic properties of laminates as an optimisation problem: a unified approach based on polar tensor invariants. Structural and Multidisciplinary Optimization 31(5), 378-387 (2005). DOI 10.1007/s00158-005-0566-5 3, 11

[8] Vannucci, P., Vincenti, A.: The design of laminates with given thermal/hygral expansion coefficients: A general approach based upon the polar-genetic method. Composite Structures 79(3), 454-466 (2007). DOI 10.1016/j.compstruct.2006.02.004 3, 11

[9] Vannucci, P., Barsotti, R., Bennati, S.: Exact optimal flexural design of laminates. Composite Structures 90(3), 337-345 (2009). DOI 10.1016/j.compstruct.2009.03.017 3,11

[10] Vincenti, A., Desmorat, B.: Optimal orthotropy for minimum elastic energy by the polar method. Journal of Elasticity 102(1), 55-78 (2010). DOI 10.1007/ s10659-010-9262-9 3, 11

[11] Vincenti, A., Vannucci, P., Ahmadian, M.R.: Optimization of laminated composites by using genetic algorithm and the polar description of plane anisotropy. Mechanics of Advanced Materials and Structures 20(3), 242-255 (2013). DOI 10.1080/15376494. $2011.5634153,11$

[12] Catapano, A., Desmorat, B., Vannucci, P.: Stiffness and strength optimization of the anisotropy distribution for laminated structures. Journal of Optimization Theory and Applications 167(1), 118-146 (2015). DOI 10.1007/s10957-014-0693-5. URL http://dx.doi.org/10.1007/s10957-014-0693-5 3,11, 14, 32, 34

[13] Jibawy, A., Julien, C., Desmorat, B., Vincenti, A., Léné, F.: Hierarchical structural optimization of laminated plates using polar representation. International Journal of Solids and Structures 48(18), 2576-2584 (2011). DOI 10.1016/j.ijsolstr.2011.05.015 3,11 
[14] Montemurro, M., Vincenti, A., Vannucci, P.: A two-level procedure for the global optimum design of composite modular structures - application to the design of an aircraft wing. part 1: theoretical formulation. Journal of Optimization Theory and Applications 155(1), 1-23 (2012). DOI 10.1007/s10957-012-0067-9 3, 11, 14, 32

[15] Montemurro, M., Vincenti, A., Vannucci, P.: A two-level procedure for the global optimum design of composite modular structures-application to the design of an aircraft wing. part 2: Numerical aspects and examples. Journal of Optimization Theory and Applications 155, 24-53 (2012) 3, 11, 14, 32

[16] Vannucci, P.: Strange laminates. Mathematical Methods in the Applied Sciences 35(13), 1532-1546 (2012). DOI 10.1002/mma.2539 3, 11

[17] Hughes, T., Cottrell, J., Bazilevs, Y.: Isogeometric analysis: CAD, finite elements, NURBS, exact geometry and mesh refinement. Computer Methods in Applied Mechanics and Engineering 194(39-41), 4135-4195 (2005). DOI 10.1016/j.cma.2004.10. 0083

[18] Bazilevs, Y., Calo, V.M., Hughes, T.J.R., Zhang, Y.: Isogeometric fluid-structure interaction: theory, algorithms, and computations. Computational Mechanics 43(1), 3-37 (2008). DOI 10.1007/s00466-008-0315-x 3

[19] Bazilevs, Y., Calo, V.M., Zhang, Y., Hughes, T.J.R.: Isogeometric fluid-structure interaction analysis with applications to arterial blood flow. Computational Mechanics 38(4), 310-322 (2006). DOI 10.1007/s00466-006-0084-3. URL https: //doi.org/10.1007/s00466-006-0084-3 3

[20] Bazilevs, Y., Hsu, M.C., Scott, M.: Isogeometric fluid-structure interaction analysis with emphasis on non-matching discretizations, and with application to wind turbines. Computer Methods in Applied Mechanics and Engineering 249-252, 28-41 (2012). DOI 10.1016/j.cma.2012.03.028 3

[21] Cho, S., Ha, S.H.: Isogeometric shape design optimization: exact geometry and enhanced sensitivity. Structural and Multidisciplinary Optimization 38(1), 53-70 (2008). DOI 10.1007/s00158-008-0266-z 3

[22] Qian, X.: Full analytical sensitivities in NURBS based isogeometric shape optimization. Computer Methods in Applied Mechanics and Engineering 199(29-32), 2059-2071 (2010). DOI 10.1016/j.cma.2010.03.005 3

[23] De Nazelle, P.: Paramétrage de formes surfaciques pour l'optimisation. Ph.D. thesis, Ecole Centrale Lyon (2013) 3

[24] Julisson, S.: Shape optimization of thin shell structures for complex geometries. Ph.D. thesis, Paris-Saclay University (2016). URL https://tel. archives-ouvertes.fr/tel-01503061 3

[25] Kpadonou, D.F.: Shape and anisotropy optimization by an isogeometric-polar approach. Ph.D. thesis, Paris-Saclay University (2017) 4, 18, 32

[26] Montemurro, M., Catapano, A.: A new paradigm for the optimum design of variable angle tow laminates. In: Variational analysis and aerospace engineering: mathematical challenges for the aerospace of the future, Springer Optimization and Its 
Applications, vol. 116, pp. 375-400. Springer (2016). URL http://dx.doi.org/10. 1007/978-3-319-45680-5 4, 14

[27] Montemurro, M., Catapano, A.: On the effective integration of manufacturability constraints within the multi-scale methodology for designing variable angle-tow laminates. Composite Structures 161, 145-159 (2017) 4, 14, 33

[28] Morgan, K.: The finite element method for elliptic problems, phillipe g. ciarlet, northholland, amsterdam, 1978. no. of pages 530. price $\$ 57.75$. International Journal for Numerical Methods in Engineering 14(5), 786-786 (1979). DOI 10.1002/nme. 16201405145

[29] Banichuk, N.V.: Introduction to Optimization of Structures. Springer New York (1990). DOI 10.1007/978-1-4612-3376-3 5

[30] Ciarlet, P.G.: An Introduction to Differential Geometry with Applications to Elasticity. Springer-Verlag (2005). DOI 10.1007/1-4020-4248-5 5

[31] Love, A.E.H.: The small free vibrations and deformation of a thin elastic shell. Philosophical Transactions of the Royal Society A: Mathematical, Physical and Engineering Sciences 179(0), 491-546 (1888). DOI 10.1098/rsta.1888.00165

[32] Koiter, W.: Foundations and Basic Equations of Shell Theory: A Survey of Recent Progress. Afdeling der Werktuigbouwkunde: WTHD. Labor. voor Techn. Mechanica (1968). URL https://books.google.fr/books?id=74REHQAACAAJ 6

[33] Naghdi, P.: Foundations of Elastic Shell Theory. North-Holland Publishing CO. Amsterdam (1963) 6, 7

[34] Reissner, E.: On the theory of transverse bending of elastic plates. International Journal of Solids and Structures 12(8), 545-554 (1976). DOI 10.1016/0020-7683(76) 90001-9 6

[35] Bézier, P.: Essai de définition numérique des courbes et des surfaces expérimentales: contribution à l'étude des propriétés des courbes et des surfaces paramétriques polynomiales à coefficients vectoriels. vol. 1 (1977). URL https://books.google.fr/ books?id=vK4POAAACAAJ 8

[36] Rogers, D.F.: An Introduction to NURBS with Historical Perspective. Elsevier (2001). DOI 10.1016/b978-1-55860-669-2.x5000-3 8, 18

[37] de Boor, C.: On the evaluation of box splines. Numerical Algorithms 5(1), 5-23 (1993). DOI 10.1007/bf02109280 9

[38] Verchery, G.: Les invariants des tenseurs d'ordre 4 du type de l'élasticité. In: J.P. Boehler (ed.) Mechanical Behavior of Anisotropic Solids / Comportment Méchanique des Solides Anisotropes, pp. 93-104. Springer Netherlands (1982). DOI 10.1007/ 978-94-009-6827-1_7 11

[39] Vannucci, P.: Plane anisotropy by the polar method. Meccanica 40, 437-454 (2005). DOI 10.1007/s11012-005-2132-z. URL http://dx.doi.org/10.1007/ s11012-005-2132-z 11, 12 
[40] Vannucci, P.: A note on the elastic and geometric bounds for composite laminates. Journal of Elasticity 112, 199-215 (2013). DOI 10.1007/s10659-012-9406-1. URL http://dx.doi.org/10.1007/s10659-012-9406-1 11, 14, 33

[41] Vannucci, P., Verchery, G.: Anisotropy of plane complex elastic bodies. International Journal of Solids and Structures 47, 1154 - 1166 (2010). DOI http: //dx.doi.org/10.1016/j.ijsolstr.2010.01.002. URL http://www.sciencedirect.com/ science/article/pii/S002076831000003X 11

[42] Valot, E., Vannucci, P.: Some exact solutions for fully orthotropic laminates. Composite Structures 69(2), 157-166 (2005). DOI 10.1016/j.compstruct.2004.06.007 11

[43] Vannucci, P., Pouget, J.: Laminates with given piezoelectric expansion coefficients. Mechanics of Advanced Materials and Structures 13(5), 419-427 (2006). DOI 10. 1080/15376490600777699 11

[44] Vannucci, P.: Influence of invariant material parameters on the flexural optimal design of thin anisotropic laminates. International Journal of Mechanical Sciences 51, 192 - 203 (2009). DOI http://dx.doi.org/10.1016/j.ijmecsci.2009.01.005. URL http://www.sciencedirect.com/science/article/pii/S0020740309000198 11, 12

[45] Vannucci, P.: A new general approach for optimizing the performances of smart laminates. Mechanics of Advanced Materials and Structures 18(7), 548-558 (2011). DOI 10.1080/15376494.2011.605015 11

[46] Montemurro, M., Koutsawa, Y., Belouettar, S., Vincenti, A., Vannucci, P.: Design of damping properties of hybrid laminates through a global optimisation strategy. Composite Structures 94(11), 3309-3320 (2012). DOI 10.1016/j.compstruct.2012.05. 00311

[47] Vannucci, P.: The design of laminates as a global optimization problem. Journal of Optimization Theory and Applications 157(2), 299-323 (2012). DOI 10.1007/ s10957-012-0175-6 11, 32

[48] Montemurro, M., Vincenti, A., Koutsawa, Y., Vannucci, P.: A two-level procedure for the global optimization of the damping behavior of composite laminated plates with elastomer patches. Journal of Vibration and Control 21(9), 1778-1800 (2013). DOI $10.1177 / 107754631350335811$

[49] Vannucci, P.: The polar analysis of a third order piezoelectricity-like plane tensor. International Journal of Solids and Structures 44(24), 7803-7815 (2007). DOI 10. 1016/j.ijsolstr.2007.05.012 11

[50] Vannucci, P.: On special orthotropy of paper. Journal of Elasticity 99(1), 75-83 (2009). DOI 10.1007/s10659-009-9232-2 11

[51] Catapano, A., Desmorat, B., Vannucci, P.: Invariant formulation of phenomenological failure criteria for orthotropic sheets and optimisation of their strength. Mathematical Methods in the Applied Sciences 35(15), 1842-1858 (2012). DOI $10.1002 / \mathrm{mma} .253011$ 
[52] Barsotti, R., Vannucci, P.: Wrinkling of orthotropic membranes: An analysis by the polar method. Journal of Elasticity 113(1), 5-26 (2012). DOI 10.1007/ s10659-012-9408-z 11

[53] Vannucci, P.: General theory of coupled thermally stable anisotropic laminates. Journal of Elasticity 113(2), 147-166 (2012). DOI 10.1007/s10659-012-9415-0 11

[54] Desmorat, B., Vannucci, P.: An alternative to the kelvin decomposition for plane anisotropic elasticity. Mathematical Methods in the Applied Sciences 38(1), 164-175 (2013). DOI 10.1002/mma.3059 11

[55] Vannucci, P., Desmorat, B.: Analytical bounds for damage induced planar anisotropy. International Journal of Solids and Structures 60-61, 96 - 106 (2015). DOI http://dx.doi.org/10.1016/j.ijsolstr.2015.02.017. URL http://www . sciencedirect.com/science/article/pii/S0020768315000578 11, 14

[56] Vannucci, P.: A note on the computation of the extrema of young's modulus for hexagonal materials: An approach by planar tensor invariants. Applied Mathematics and Computation 270, 124-129 (2015). DOI 10.1016/j.amc.2015.08.025 11

[57] Vannucci, P., Desmorat, B.: Plane anisotropic rari-constant materials. Mathematical Methods in the Applied Sciences 39(12), 3271-3281 (2015). DOI 10.1002/mma.3770 11

[58] Vannucci, P.: A special planar orthotropic material. Journal of Elasticity 67, 8196 (2002). DOI 10.1023/A:1023949729395. URL http://dx.doi.org/10.1023/A: 102394972939512

[59] Vannucci, P., Verchery, G.: Stiffness design of laminates using the polar method. International Journal of Solids and Structures 38(50-51), 9281 - 9294 (2001). DOI http: //dx.doi.org/10.1016/S0020-7683(01)00177-9. URL http://www. sciencedirect. $\mathrm{com} / \mathrm{science/article/pii/S0020768301001779} 13$

[60] de Boor, C.: A Practical Guide to Splines. Applied Mathematical Sciences. Springer, New York (2001). URL https://books.google.fr/books?id=m0QDJvBI_ecC 15, 18

[61] Patrikalakis, N.M., Maekawa, T.: Shape interrogation for computer aided design and manufacturing. Springer, Berlin (2009) 18

[62] Hooke, R.: A description of helioscopes, and some other instruments. John and Martyn Printer, London, UK (1675) 23

[63] Heyman, J.: The stone skeleton. Cambridge University Press, Cambridge, UK (1995) 23

[64] Cowan, H.J.: The masterbuilders. Wiley, New York, USA (1977) 23 